\title{
Espaces d'arcs et invariants d'Alexander
}

\author{
Gil Guibert
}

Résumé. Nous calculons la fonction zeta d'Igusa motivique de Denef-Loeser associée à une série irréductible de deux variables et retrouvons à l'aide de ce résultat la formule donnant le spectre de Hodge-Steenbrink d'une courbe plane irréductible en termes des données de Puiseux. Nous étudions ensuite une généralisation de la fonction d'Igusa à une famille de fonctions et montrons que cette fonction d'Igusa permet de retrouver les invariants d'Alexander de la famille. Nous appliquons ce résultat en dimension deux pour obtenir une expression du polynôme d'Alexander d'une courbe plane.

Abstract. We compute the motivic Igusa zeta function of Denef-Loeser associated with a two variables irreducible serie and use this result to give a new proof of the formula expressing the Hodge-Steenbrink spectrum in terms of the Puiseux data. We study a generalisation of the motivic Igusa function to a family of functions and show that this Igusa function is related with the Alexander invariants of the family. Using this result, we obtain a formula for the Alexander plolynomial of a plane curve.

Mathematics Subject Classification (2000). 14B05, 14H20, 13D40.

Mots clés. Espaces d'arcs, fonction d'Igusa motivique, fibre de Milnor, singularités de courbe plane, invariants d'Alexander.

Keywords. Arcs spaces, motivic Igusa function, plane curve singularity, Alexander invariants.

\section{Introduction}

Le point de départ de ce travail est la fonction zêta d'Igusa motivique introduite par Denef et Loeser dans [11]. Sa définition utilise la théorie de l'intégration motivique, introduite par Kontsevitch [18] et développée par Batyrev et DenefLoeser, qui est un analogue sur $k((t))$ ( $k$ désigne un corps de caractéristique nulle) de l'intégration $p$-adique. Dans cette théorie, $\mathbf{Q}_{p}$ est remplacé par $k((t))$ et l'anneau $\mathbf{Z}_{p}$ par $k[[t]]$. L'espace d'intégration n'est plus l'espace des points entiers d'une variété définie sur un corps $p$-adique mais l'espace des arcs tracés sur la variété : c'est un schéma noté $\mathcal{L}(X)$ dont les points $k$-rationnels sont les points $k[[t]$-rationnels de $X$. Plus précisemment, on peut, pour tout entier naturel $n$ définir le schéma des arcs tronqués à l'ordre $n$ tracés dans $X$, noté $\mathcal{L}_{n}(X)$ dont les points $k$-rationnels sont les points $k[[t]] /\left(t^{n+1}\right)$-rationnels de $X$; si $m \geq n$, on 
dispose d'un morphisme naturel de troncation $\pi_{m, n}: \mathcal{L}_{m}(X) \rightarrow \mathcal{L}_{n}(X)$; on définit alors $\mathcal{L}(X)$ comme la limite projective des $\mathcal{L}_{n}(X)$ dans la catégorie des schémas. Notons que $\mathcal{L}_{0}(X) \simeq X$, ce qui permet de voir $\mathcal{L}_{n}(X)$ comme variété au-dessus de $X$ via le morphisme $\pi_{n, 0}$. Les intégrales considérées sont à valeurs dans un anneau $\hat{\mathcal{M}}_{k}$ défini de la façon suivante :

On considère le groupe de Grothendieck $K_{0}\left(\operatorname{Var}_{k}\right)$ des variétés : c'est le groupe engendré par les classes d'isomorphisme de variétés sur $k$ et les relations $[X]=$ $[Y]+[X \backslash Y]$ si $Y$ est un fermé de Zariski de $X$. On note $\mathbb{L}:=\left[\mathbf{A}_{k}^{1}\right]$ et on définit $\mathcal{M}_{k}$ comme le localisé en $\mathbb{L}$ de $K_{0}\left(\operatorname{Var}_{k}\right) ; \hat{\mathcal{M}}_{k}$ est défini comme le complété de $\mathcal{M}_{k}$ pour la filtration par la dimension (voir par exemple [15] et [20]). La fonction zêta d'Igusa motivique est un analogue dans le contexte de l'intégration motivique de la fonction zêta d'Igusa locale associée à un polynôme (voir [7] à ce sujet).

Cette fonction est définie comme suit :

Soit $X$ une variété algébrique complexe de dimension $d, x$ un point de $X$; on définit $X_{n}$ comme le sous-espace de $\pi_{n, 0}^{-1}(\{x\})$ sur lequel $f$ est d'ordre $n$. On dispose d'un morphisme naturel de $X_{n}$ vers le groupe multiplicatif qui à un $\operatorname{arc} \varphi$ associe le coefficient de $t^{n}$ dans la série $f(\varphi)$; on le note $a c(f)$. La fibre $X_{n, 1}$ au-dessus de 1 de la restriction de $a c(f)$ à $X_{n}$ est naturellement munie d'une action du groupe des racines $n$-ièmes de l'unité induite par multiplication sur le paramètre $t$, ce qui permet de définir la classe de $X_{n, 1}$ dans l'anneau $\mathcal{M}_{k}^{\hat{\mu}}$ (qui est une version de $\mathcal{M}_{k}$ pour les variétés munies d'une action du pro-groupe $\hat{\mu}$ des racines de l'unité). On pose alors

$$
Z_{f, x}(T):=\sum_{n \geq 1}\left[X_{n, 1}, \hat{\mu}\right] \mathbb{L}^{-n d} T^{n}
$$

Inspirés par des travaux de Denef dans le cadre $p$-adique, Denef et Loeser ont exploré les rapports entre la fonction d'Igusa motivique et les invariants classiques associés au faisceau des cycles évanescents de $f$ tels que la fonction zêta de la monodromie et le spectre de Hodge. Ils ont montré que la fibre de Milnor motivique, qui est une limite en $+\infty$ de la fonction d'Igusa motivique, et la fibre de Milnor usuelle ont même réalisation de Hodge [11].

Il devient alors naturel de tenter de décrire les espaces d'arcs afin d'obtenir des formules explicites pour certains invariants locaux des singularités de fonction. C'est la motivation de ce travail.

L'objet du premier paragraphe est d'introduire les outils nécessaires à la définition de la fonction d'Igusa motivique et de rappeler un résultat fondamental de Denef-Loeser sur le lien entre cette fonction et la théorie des cycles évanescents. Nous terminons par le calcul de la fibre de Milnor motivique dans le cas d'une fonction non dégénérée par rapport à son polygone de Newton.

Le paragraphe suivant est consacrée à l'étude des espaces d'arcs associés à une série irréductible de deux variables. Nous décrivons ces espaces à l'aide de deux paramètres : la valuation en $t$ d'une coordonnée transverse et le contact de l'arc avec les développements de Puiseux de la courbe. Cette description aboutit au calcul de la fibre de Milnor motivique de $f$ à l'aide des paires de Puiseux (Théorème 3.3.1). 
En utilisant le théorème de Denef-Loeser mentionné plus haut, nous retrouvons à partir de cet énoncé la formule exprimant le spectre de Hodge en termes des données de Puiseux (cf. corollaire 3.4.1).

Nous nous intéressons au troisième paragraphe à une généralisation naturelle de la fonction d'Igusa motivique à plusieurs fonctions $f_{1}, \ldots f_{p}$. Si $X_{0}:=\cap_{i=1}^{p} f_{i}^{-1}(0)$, cette fonction est définie comme une série à coefficients dans un anneau de variétés relatives $\mathcal{M}_{X_{0} \times \mathbf{G}_{m, \mathbf{C}}^{p}}$. Dans l'article [21], Sabbah généralise à une famille de fonctions $f_{1}, \ldots, f_{p}$ la construction du complexe des cycles proches associé à une fonction analytique. Ainsi, pour tout point $x$ de $X_{0}$, il associe à cette famille de fonctions sa fonction zêta d'Alexander en $x$ notée ${ }^{A} \zeta_{f, x}$; c'est une fraction rationnelle à $p$ variables qui coïncide avec l'inverse de la fonction zêta de la monodromie lorsque $p=1$ et avec le polynôme d'Alexander de l'entrelac algébrique associé à la courbe d'équation $f_{1} \ldots f_{p}$ lorsque l'espace ambiant est de dimension deux et les $f_{i}$ sont des séries irréductibles deux à deux distinctes.

Une question naturelle est l'étude du lien entre cette fonction d'Alexander et la fonction d'Igusa motivique définie précédemment. Nous donnons un énoncé qui montre que la première se déduit de la seconde (Théorème 4.4.1).

Nous nous intéressons ensuite au calcul de la fonction d'Igusa motivique associée à une famille de $p$ séries irréductibles de deux variables. Nous représentons les différents développements de Puiseux de ces fonctions sur un arbre qui reflète les contacts mutuels de ces développements. La fonction d'Igusa motivique associée s'exprime comme une combinaison linéaires à coefficients dans $\mathcal{M}_{X_{0} \times \mathbf{G}_{m, \mathbf{C}}^{p}}$ de fractions rationnelles à $p$ variables, indexée par les sommets, branches et feuilles de l'arbre des contacts. En utilisant le théorème 4.4.1, on en déduit une formule exprimant la fonction zêta d'Alexander des $p$ fonctions en termes de l'arbre des contacts (proposition 5.3.1).

Nous terminons en appliquant les résultats précédents au calcul de la fibre de Milnor motivique dans le cas d'une fonction de deux variables non nécessairement irréductible (proposition 5.4.1).

Remerciements. L'auteur tient à remercier M. Merle sans les conseils et les encouragements duquel ce travail n'aurait pas vu le jour, N. A'Campo et F. Loeser pour d'intéressantes discussions, ainsi que M. Brion dont les remarques et suggestions ont contribué à améliorer ce texte.

\section{Fonction zêta d'Igusa motivique associée à une fonction régu- lière et fibre de Milnor}

Les deux références principales pour cette section sont [15] et [20].

Dans ce paragraphe $k$ désignera un corps algébriquement clos de caractéristique nulle. 
Espaces d'arcs associés à une variété algébrique. Soit $X$ une variété algébrique de dimension $d$ sur $k$. Pour tout entier $n$, on peut définir le schéma $\mathcal{L}_{n}(X)$ des germes d'arcs tronqués à l'ordre $n$ sur $X$, dont les points $K$-rationnels sont les points $K[[t]] / t^{n+1} K[[t]]$ rationnels de $X$ (pour tout corps $K$ contenant $k$ ). Si $n \geq m$, on dispose d'un morphisme naturel de troncation $\pi_{n, m}: \mathcal{L}_{n}(X) \rightarrow$ $\mathcal{L}_{m}(X)$. Les $\mathcal{L}_{n}(X)$ forment un système projectif pour les morphismes $\pi_{n, m}$. On note $\mathcal{L}(X)$ la limite projective de ce système dans la catégorie des schémas. C'est un schéma dont les points $K$-rationnels sont les points $K[[t]]$-rationnels de $X$ (pour tout corps $K$ contenant $k$ ). Notons que $\mathcal{L}_{0}(X)=X$ et qu'on dispose donc d'un morphisme naturel $\pi_{0}: \mathcal{L}(X) \rightarrow X$ qui à un arc associe son origine. Si $X$ est lisse, pour tout entier naturel $n, \mathcal{L}_{n}(X)$ est un fibré en espaces affines de dimension $n d$ au-dessus de $X$. Notons que les définitions précédentes s'étendent au cas où $X$ est un schéma formel.

Groupes de Grothendieck de variétés. Soit $S$ une variété algébrique sur $k$. On appelle $S$-variété une variété algébrique $X$ sur $k$ munie d'un morphisme $X \rightarrow S$ et on note $\operatorname{Var}_{S}$ la catégorie dont les objets sont des $S$-variétés et les morphismes sont les morphismes de $k$-variété commutant aux morphismes vers $S$. On notera $X / S$ une $S$-variété ou seulement $X$ lorsque le contexte sera clair. On définit le groupe de Grothendieck relatif $K_{0}\left(\operatorname{Var}_{S}\right)$ des $S$-variétés comme le groupe engendré par les classes d'isomorphismes de $S$-variétés $[X]$ (pour $X$ une $S$-variété) et quotienté par les relations $[X]=[Y]+[X \backslash Y]$ si $Y$ est un fermé de Zariski de $X$. Le produit fibré au-dessus de $S$ munit $K_{0}\left(\operatorname{Var}_{S}\right)$ d'une structure d'anneau. Par ailleurs, on peut associer à tout ensemble constructible, c'est-à-dire à toute réunion finie de variétés au-dessus de $S$ localement fermées, sa classe dans $K_{0}\left(\operatorname{Var}_{S}\right)$ : si $\mathcal{X}=\cup_{i=1}^{n} X_{i}$ où les $X_{i}$ désignent des variétés localement fermées, on note $[\mathcal{X}]=\sum_{i=1}^{n}\left[X_{i}\right]-\sum_{1 \leq i<j \leq n}\left[X_{i} \cap X_{j}\right]+\ldots+(-1)^{n-1}\left[\cap_{i=1}^{n} X_{i}\right]$ (cet élément de $K_{0}\left(\operatorname{Var}_{S}\right)$ est indépendant du choix des $\left.X_{i}\right)$.

On note $\mathbb{L}$ la classe de $\mathbf{A}_{k}^{1} \times S$ (vue comme S-variété via la projection naturelle sur $S$ ) dans $K_{0}\left(\operatorname{Var}_{S}\right)$ et on définit $\mathcal{M}_{S}$ comme l'anneau obtenu par localisation de $K_{0}\left(\operatorname{Var}_{S}\right)$ en $\mathbb{L}$.

Si $S=\operatorname{Spec}(k)$, on adoptera la notation $\operatorname{Var}_{k}$ pour $\operatorname{Var}_{S}$. L'application naturelle $X \rightarrow[X]$ de $\operatorname{Var}_{k}$ dans $K_{0}\left(\operatorname{Var}_{k}\right)$ s'interprète comme la caractéristique d'Euler universelle. Notons que si $s$ est un point de $S$ on dispose d'une application naturelle Fibre $_{s}$ de $K_{0}\left(\operatorname{Var}_{S}\right)$ dans $K_{0}\left(\operatorname{Var}_{k}\right)$ définie par $[X] \mapsto\left[X_{s}\right], X_{s}$ désignant la fibre de $X \rightarrow S$ au-dessus de $s$.

Ces définitions peuvent s'étendre au cas de variétés munies de l'action d'un groupe de racines de l'unité de la manière suivante : pour tout $n \in \mathbf{N}$ on note $\mu_{n}$ le groupe des racines $n$-ièmes de l'unité; les $\mu_{n}$ forment un système projectif pour les applications $x \mapsto x^{d}$ de $\mu_{n d}$ dans $\mu_{n}$; on note $\hat{\mu}$ la limite projective de ce système. Une action de $\mu_{n}$ sur une $S$-variété $X$ est dite bonne si toute orbite est contenue dans une sous-variété affine de $X$. On définit le groupe de Grothendieck monodromique $K_{0}^{\hat{\mu}}\left(\operatorname{Var}_{S}\right)$ comme l'analogue de $K_{0}\left(\operatorname{Var}_{S}\right)$ pour les variétés munies d'une bonne action de $\hat{\mu}$. Si $X$ est une variété munie d'une bonne action de $\hat{\mu}$, on 
notera parfois $[X, \hat{\mu}]$ la classe de $X$ dans $K_{0}^{\hat{\mu}}\left(\operatorname{Var}_{S}\right)$.

Mesure motivique. Un sous-ensemble $S$ de $\mathcal{L}(X)$ est dit stable si il existe un entier naturel $n$ et un sous-espace constructible $C$ de la variété $\mathcal{L}_{n}(X)$ tel que $S=\pi_{n}^{-1}(C)$ et tel que pour tout $m$ supérieur à $n$, la restriction de $\pi_{m, n}$ à $S$ soit une fibration triviale par morceaux en espaces affines. La deuxième condition est automatiquement vérifiée si $X$ est lisse. Si $S$ est stable, la quantité $\left[\pi_{m}(S)\right] \mathbb{L}^{-(m+1) d}$ est indépendante de $m$ pour $m$ assez grand. On la note $\mu(S)$. L'application $\mu$ définit une mesure, appelée mesure motivique, sur la sous-algèbre booléenne des espaces stables de $\mathcal{L}(X)$. Notons que cette mesure peut s'étendre en une mesure $\tilde{\mu}$ définie sur une algèbre de sous-ensembles de $\mathcal{L}(X)$ (qui est par définition l'algèbre des parties mesurables) et à valeurs dans le complété de $\mathcal{M}_{S}$ pour une filtration convenable. Nous n'utiliserons pas cette généralisation dans cet article.

Caractéristique de Hodge monodromique et spectre de Hodge. Dans la suite, on travaillera sur le corps des complexes. On appelle structure de Hodge la donnée d'un Q-espace vectoriel de dimension finie $H$ muni d'une bigraduation $H \otimes$ $\mathbf{C}=\oplus_{p, q \in \mathbf{Z}} H^{p, q}$ telle que $H^{p, q}=\overline{H^{q, p}}$ et que pour tout $m$, l'espace $\oplus_{p+q=m} H^{p, q}$ homogène de poids $m$, soit défini sur $\mathbf{Q}$; en considérant les morphismes d'espaces vectoriels respectant la bigraduation, on obtient une catégorie abélienne notée HS munie d'un produit tensoriel. On peut alors définir le groupe de Grothendieck associé $K_{0}(\mathrm{HS})$ qui est naturellement muni d'une structure d'anneau.

Une structure de Hodge mixte est la donnée d'un Q-espace vectoriel de dimension finie $V$ muni d'une filtration croissante finie $W \cdot V$ (appelée filtration par le poids) telle que $G r_{\bullet}^{W}(V)$ soit munie d'une structure de Hodge dont la composante homogène de degré $m$ est $G r_{m}^{W}(V)$. Le groupe de Grothendieck de la catégorie ainsi obtenue est aussi égal à $K_{0}(\mathrm{HS})$ et la classe de $V$ dans $K_{0}(\mathrm{HS})$ vaut $[V]=\sum_{m}\left[G r_{m}^{W}(V)\right]$.

Pour toute variété algébrique $X$ sur $\mathbf{C}$, les groupes de cohomologie à support compact $H_{c}^{i}(X, \mathbf{Q})$ admettent une structure de Hodge mixte. On peut donc associer à toute variété $X$ sa caractéristique de Hodge dans $K_{0}(\mathrm{HS}): \chi_{h}(X):=$ $\sum_{i}(-1)^{i}\left[H_{c}^{i}(X, \mathbf{Q})\right]$ où $\left[H_{c}^{i}(X, \mathbf{Q})\right]$ est la classe de $H_{c}^{i}(X, \mathbf{Q})$ dans $K_{0}(\mathrm{HS})$. Si $Y$ est une sous-variété de $X$, la suite exacte longue induite en cohomologie est compatible avec les structures de Hodge correspondantes, si bien que la caractéristique de Hodge $\chi_{h}$ s'étend en un morphisme d'anneaux de $K_{0}\left(\operatorname{Var}_{\mathbf{C}}\right)$ dans $K_{0}(\mathrm{HS})$ que l'on note encore $\chi_{h}$. L'image de la droite affine étant inversible dans $K_{0}(\mathrm{HS})$, on en déduit que $\chi_{h}$ s'étend à $\mathcal{M}_{\mathbf{C}}$.

Notons $\mathrm{HS}^{\mathrm{mon}}$ la catégorie abélienne des structures de Hodges mixtes munies de l'action d'un endomorphisme quasi-unipotent. Si $X$ est une variété algébrique munie d'une bonne action de $\hat{\mu}$, ses groupes de cohomologie sont munies de l'action d'un endomorphisme quasi-unipotent; ceci permet de définir la caractéristique de Hodge monodromique de $X$

$$
\chi_{h}^{\operatorname{mon}}(X)=\sum_{i}(-1)^{i}\left[H_{c}^{i}(X, \mathbf{Q})\right] \in K_{0}\left(\mathrm{HS}^{\mathrm{mon}}\right) .
$$


Par ailleurs, on sait associer à un élément de $\mathrm{HS}^{\text {mon }}$ son spectre de Hodge ; pour $\alpha \in \mathbf{Q}$, on note $H_{\alpha}^{p, q}$ l'espace caractéristique de $H^{p, q}$ associé à la valeur propre $\exp (2 \pi i \alpha)$. Le spectre de Hodge de $H$ est le polynôme de Laurent en $t^{\frac{1}{m}}$, (où $m$ désigne l'ordre de quasi-unipotence de l'automorphisme correspondant), défini par :

$$
h \operatorname{sp}(H):=\sum_{\alpha \in \mathbf{Q} \cap[0,1[} t^{\alpha}\left(\sum_{p, q \in \mathbf{Z}} t^{p} \operatorname{dim}\left(H_{\alpha}^{p, q}\right)\right) .
$$

On note $S p$ la composée de $h s p: K_{0}\left(\mathrm{HS}^{\mathrm{mon}}\right) \rightarrow \cup_{n \geq 1} \mathbf{Z}\left[t^{1 / n}, t^{-1 / n}\right]$ et de la caractéristique de Hodge monodromique $\chi_{h}^{\text {mon }}$.

Fonction zeta d'Igusa motivique. Dans cette section, on désigne par $f$ : $X \rightarrow \mathbb{A}_{k}^{1}$ une fonction régulière et on pose $X_{0}=f^{-1}(0)$.

Dans cette situation, si $x$ est un point de $X_{0}$, on dispose d'un certain nombre d'invariants classiques liés à la la fibration de Milnor associée à $f$ et à l'action de la monodromie sur la cohomologie de la fibre, comme la fonction zêta de la monodromie ou le spectre de Hodge ([23] et [24]). L'objet de cette section est d'énoncer un résultat de Denef-Loeser sur le lien entre ces invariants et la fonction d'Igusa motivique associée à $f$. On notera ord $t$ la valuation $t$-adique d'une série de la variable $t$.

Pour tout entier $n$, on définit suivant Denef-Loeser

$$
X_{n}:=\left\{\varphi \in \mathcal{L}_{n}(X), \operatorname{ord}_{t} f \circ \varphi=n\right\} .
$$

Notons que ce sous-espace constructible de $\mathcal{L}_{n}(X)$ est naturellement muni d'une action de $\mathbf{G}_{m, \mathbf{C}}$ définie par $(c . \varphi)(t):=\varphi(c t)$; cette action induit clairement une bonne action de $\mu_{n}$ sur la fibre $X_{n, 1}:=\left\{\varphi \in X_{n} \mid a c(f)(\varphi)=1\right\}$.

Ceci permet de définir la fonction zêta motivique associée à $f$ comme la série formelle à coefficients dans $\mathcal{M}_{X_{0}}^{\hat{\hat{}}}$ :

$$
Z_{f}(T):=\sum_{n \geq 1}\left[X_{n, 1} / X_{0}, \hat{\mu}\right]\left(\mathbb{L}^{-d} T\right)^{n} .
$$

Un résultat de Denef-Loeser (voir par exemple [15] Théorème 3.3.1) exprimant $Z_{f}(T)$ à l'aide d'une résolution des singularités montre que $Z_{f}(T)$ est un élément de la sous $\mathcal{M}_{X_{0}}^{\hat{\mu}}$-algèbre de $\mathcal{M}_{X_{0}}^{\hat{\mu}}[[T]]$ engendrée par les fractions rationnelles du type $\frac{\mathbb{L}^{-a} T^{b}}{\mathbb{L}^{-a} T^{b}-1}$ (pour $a$ et $b$ entiers naturels).

Notons qu'on peut définir un morphisme $\lim _{T \rightarrow \infty}$ de cette sous-algèbre dans $\mathcal{M}_{X_{0}}^{\hat{\mu}}$ en posant $\lim _{T \rightarrow \infty}\left(\frac{\mathbb{L}^{-a} T^{b}}{1-\mathbb{L}^{-a} T^{b}}\right)=-1$.

Ceci permet de définir un élément $\mathcal{S}_{f}:=-\lim _{T \rightarrow \infty} Z_{f}(T)$ dans $\mathcal{M}_{X_{0}}^{\hat{\mu}}$. On pose $\mathcal{S}_{f, x}:=\operatorname{Fibre}_{x}\left(\mathcal{S}_{f}\right)$ pour $x \in X_{0}$; cette variété virtuelle est par définition la fibre de Milnor motivique de $f$ en $x$.

On note par ailleurs $\mathcal{S}_{f}^{\Phi}:=(-1)^{d-1}\left(\mathcal{S}_{f}-\left[X_{0}\right]\right)$.

Indiquons un résultat de Denef et Loeser qui montre que $\mathcal{S}_{f}^{\Phi}$ est lié au complexe de faisceaux des cycles évanescents de $f$ sur $X_{0}$. 
Les groupes de cohomologie à support compact de la fibre de Milnor $F_{x}$ d'une fonction $f$ en $x \in f^{-1}(0)$ sont munis d'une structure de Hodge mixte naturelle compatible avec l'action de l'endomorphisme quasi-unipotent de monodromie $M_{x}$ (voir [23]).

On définit alors le spectre de Hodge-Steenbrink de $f$ en $x$ par :

$$
h s p(f, x):=(-1)^{d} h s p\left(\chi_{h}^{\operatorname{mon}}\left(F_{x}\right)-1\right) .
$$

Nous pouvons alors énoncer le théorème suivant :

Theorem 2.0.1 ([11]). La caractéristique de Hodge de la fibre de Milnor motivique et celle de la fibre de Milnor usuelle sont égales :

$$
\chi_{h}\left(\mathcal{S}_{f, x}\right)=\chi_{h}\left(F_{x}\right) .
$$

En particulier, $h s p(f, x)=S p\left(\mathcal{S}_{f, x}^{\Phi}\right)$. La fonction d'Igusa motivique associée à $f$ détermine donc le spectre de Hodge-Steenbrink de $f$ au point $x$.

Remarque 2.0.2. Dans le cas où $f: X_{x} \rightarrow \mathbf{C}, 0$ est un germe d'application analytique complexe, il est possible de définir de la même manière que ci-dessus une fonction d'Igusa motivique associée à $f$ en $x$. Les espaces $X_{n}$ utilisés dans la définition de $Z_{f}$ sont des variétés algébriques au-dessus de $X_{0}$ (pour tout $x \in X$, $X_{n} \cap \pi_{0}^{-1}(\{x\})$ ne dépend que du jet à l'ordre $n$ de $f$ en $\left.x\right)$. On peut vérifier que le théorème précédent reste vrai dans ce contexte.

\subsection{Un exemple : fonctions non dégénérées par rapport à leur polyèdre de Newton}

On s'intéresse dans cette section au calcul de la fonction zêta associée à une fonction non dégénérée par rapport à son polyèdre de Newton. On se donne donc un polynôme nul à l'origine $f: \mathbf{C}^{d} \rightarrow \mathbf{C}$ et des coordonnées $x_{1}, \ldots, x_{d}$. On note $M$ le réseau correspondant aux monômes (pour ces coordonnées) et $N$ son dual ; on note $\Gamma \subset M$ le polyèdre de Newton de $f$ pour $x_{1}, \ldots, x_{d}$ et $m_{\Gamma}$ sa fonction d'appui, c'est-à-dire la fonction qui à un élément de $N$ associe le minimum de sa restriction à $\Gamma$.

A chaque face $\gamma$ de codimension 1 de $\Gamma$ correspond un vecteur de $N$ qu'on nommera normale à $\gamma$ : c'est l'unique vecteur primitif (c'est-à-dire à coordonnées premières entre elles) de $N$ dont la restriction à $\Gamma$ atteint son minimum sur $\gamma$; on le notera $e^{(\gamma)}$. Si $a \in \mathbf{N}^{d}$, on note $\gamma(a)$ la face de $\Gamma$ sur la quelle la restriction de $a$ à $\Gamma$ atteint son minimum. L'ensemble $\left\{a \in \mathbf{N}^{d} \mid \gamma(a)=\gamma\right\}$ est un cône convexe relativement ouvert du réseau $N$; si $\gamma$ est l'intersection des faces $\gamma_{1}, \ldots, \gamma_{r}$ de codimension un, ce cône est engendré par les normales $e^{\left(\gamma_{i}\right)}$ correspondantes; on le notera $\sigma(\gamma)$. La collection de cônes ainsi définie s'organise en un éventail, qui est par définition l'éventail normal de $\Gamma$. On note $f(x):=\sum_{\nu \in\left(\mathbf{N}^{\times}\right)^{d}} f_{\nu} x^{\nu}$. Si $\gamma$ est une face de $\Gamma$, on notera $f_{\gamma}$ le polynôme quasi-homogène $\sum_{\nu \in \gamma} f_{\nu} x^{\nu}$. 
On introduit les notations suivantes : Si $\gamma$ est une face de $\Gamma$, on note $\mathcal{X}_{\gamma}(1)$ la variété $\left\{x \in \mathbf{G}_{m, \mathbf{C}}^{d} \mid f_{\gamma}(x)=1\right\}$ munie de l'action naturelle de $\mu_{m_{\Gamma}(a)}$ induite par la quasi-homogénéité de $f_{\gamma}$ et $\mathcal{X}_{\gamma}(0):=\left\{x \in \mathbf{G}_{m, \mathbf{C}}^{d} \mid f_{\gamma}(x)=0\right\}$

On suppose dorénavant que $f$ est non dégénérée par rapport à $\Gamma$ (au sens de [18]) et on cherche à calculer $Z_{f}(T) \in \mathcal{M}_{\mathbf{C}}^{\hat{\mu}}[[T]]$. On identifiera dans tout ce qui suit $\mathcal{L}\left(\mathbf{A}_{\mathbf{C}}^{d}\right)$ à $\mathbf{C}[[t]]^{d}$ via le système de coordonnées $x_{1}, \ldots, x_{d}$. Pour tout $\varphi \in \mathcal{L}\left(\mathbf{A}_{\mathbf{C}}^{d}\right)$, on note $\operatorname{ord}_{t} x(\varphi):=\left(\operatorname{ord}_{t}\left(x_{1}(\varphi)\right), \ldots, \operatorname{ord}_{t}\left(x_{d}(\varphi)\right)\right.$. Pour tout $a \in \mathbf{N}^{d}$ et $n \in \mathbf{N}$ on pose : $X_{a}:=\left\{\varphi \in \mathcal{L}\left(\mathbf{A}^{d}\right) \mid \operatorname{ord}_{t} x(\varphi)=a\right\}$ et $X_{a, n}:=X_{a} \cap X_{n, 1}$. Notons que pour tout $a \in \mathbf{N}^{d}$, et $\varphi \in X_{a}$, on a $\operatorname{ord}_{t} f(\varphi) \geq m_{\Gamma}(a)$. Pour calculer $\mu\left(Z_{n}\right)$, on peut se contenter de calculer $\sum_{a \in\left(\mathbf{N}^{*}\right)^{d}} \mu\left(X_{a, n}\right)$ car le complémentaire de $\cup_{a \in\left(\mathbf{N}^{*}\right)^{d}} X_{a, n}$ dans $X_{n}$ est de mesure motivique nulle. En utilisant le fait que les cônes de $\Sigma$ forment une partition de $\left(\mathbf{N}^{\times}\right)^{d}$, on en déduit que la fonction zêta motivique associée à $f$ s'écrit

$$
\begin{aligned}
Z_{f}(T) & =\mathbb{L}^{-d} \sum_{n \geq 1} \mu\left(X_{n, 1}\right) T^{n}=U^{-d} \sum_{\gamma} \sum_{\gamma(a)=\gamma} \sum_{n \geq m_{\Gamma}(a)} \mu\left(X_{a, n}\right) T^{n} \\
& =\mathbb{L}^{-d} \sum_{\gamma} \sum_{\gamma(a)=\gamma} \mu\left(X_{a, m_{\Gamma}(a)}\right) T^{m_{\Gamma}(a)}+\mathbb{L}^{-d} \sum_{n \geq m_{\Gamma}(a)+1} \mu\left(X_{a, n}\right) T^{n} \\
& :=\mathbb{L}^{-d}\left(\sum_{\gamma} Z_{\gamma}(T)+Z_{\gamma}^{>}(T)\right) .
\end{aligned}
$$

On peut évaluer la mesure de l'espace $Z_{a, n}$ pour $n \geq m_{\Gamma}(a)+1$ par la formule suivante :

Lemme 2.1.1. Pour tout $a \in \mathbf{N}^{d}$ et $k \geq 1$, on $a$ :

$$
\mu\left(X_{a, m_{\Gamma(a)}+k}\right)=\mathbb{L}^{-k-s(a)}\left[\mathcal{X}_{\gamma}(0)\right](\mathbb{L}-1) .
$$

Preuve. Par additivité de $\mu$, il nous suffit d'évaluer la mesure de $X_{a}^{\geq m_{\Gamma}(a)+k+1}$.

Soit $\varphi(t):=\left(x_{1}(t), \ldots, x_{d}(t)\right)$ un arc tronqué vérifiant

$$
\operatorname{ord}_{t} x(\varphi)=a \text { et } \operatorname{ord}_{t} f_{\gamma(a)}(\varphi) \geq m_{\Gamma(a)}+k .
$$

On peut donc poser $\tilde{\varphi}:=\left(t^{-a_{1}} x_{1}(t), \ldots, t^{-a_{d}} x_{d}(t)\right)$ où pour tout $i, \tilde{x}_{i}:=t^{-a_{i}} x_{i}$ est de valuation nulle. Nous pouvons écrire $f(\varphi)(t)=t^{m_{\Gamma(a)}} \tilde{f}_{\gamma(a)}(\tilde{\varphi}, t)$. Nous sommes ainsi ramenés à évaluer la mesure de $\left\{\tilde{\varphi} \mid \operatorname{ord}_{t} \tilde{f}_{\gamma(a)}(\tilde{\varphi}) \geq k+1\right\}$

Notons que la fonction $\tilde{f}_{\gamma(a)}$ est lisse en $\tilde{\varphi}(0)$ : cela résulte de l'identité

$$
\frac{\partial \tilde{f}_{\gamma(a)}}{\partial \tilde{x}_{i}}(\tilde{\varphi}(0), 0)=\frac{\partial f_{\gamma(a)}}{\partial x_{i}}(\tilde{\varphi}(0)),
$$

de l'hypothèse de non-dégénérescence et du fait que $\tilde{\varphi}(0) \in \mathbf{G}_{m, \mathbf{C}}^{d}$.

On en déduit que l'espace des solutions de $\tilde{f}_{\gamma(a)}=0\left[t^{k+1}\right]$ est un fibré audessus de $\mathcal{X}_{\gamma}(0)$ de fibre isomorphe à un espace affine de codimension $k$ dans

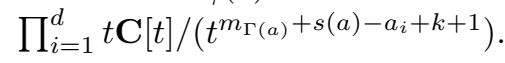


L'espace $X_{a}^{\geq m_{\Gamma}(a)+k+1}$ est donc isomorphe à $\mathcal{X}_{\gamma(a)}(0) \times \mathbf{A}^{m_{\Gamma(a)}+s(a)+(k+1) d-s(a)-k}$; sa mesure vaut donc $\left[\mathcal{X}_{\gamma(a)}(0)\right] \mathbb{L}^{-k-s(a)}$. En calculant de manière analogue la mesure de $X_{a}^{\geq m_{\Gamma}(a)+k}$, on aboutit au résultat annoncé.

Si on pose $S_{\gamma}(\mathbb{L}, T):=\sum_{a \in \sigma(\gamma)} \mathbb{L}^{-s(a)} T^{m_{\Gamma}(a)}$, on en déduit que

$$
Z_{\gamma}^{>}(T)=(\mathbb{L}-1) \frac{\mathbb{L}^{-1} T}{1-\mathbb{L}^{-1} T}\left[\mathcal{X}_{\gamma}(0)\right] S_{\gamma}(\mathbb{L}, T)
$$

d'où

$$
Z^{>}(T)=\sum_{\gamma}(\mathbb{L}-1)\left[\mathcal{X}_{\gamma}(0)\right] \frac{\mathbb{L}^{-1} T}{1-\mathbb{L}^{-1} T} S_{\gamma}(\mathbb{L}, T)
$$

Par ailleurs, on a la formule suivante :

Lemme 2.1.2. $\mu\left(X_{a, m_{\Gamma}(a)}\right)=\left[\mathcal{X}_{\gamma(a)}(1), \mu_{m_{\Gamma}(a)}\right] \mathbb{L}^{-s(a)}$.

Preuve. On fixe une forme linéaire $a$ qui atteint son minimum sur $\gamma$. L'espace $\pi_{m_{\Gamma}(a)}\left(X_{a, m_{\Gamma}(a)}\right)$ est paramétré par le produit du tore de dimension $d$ par un espace affine. On note $c=\left(c_{i}\right)_{1 \leq i \leq d}$ des coordonnées sur le premier facteur ; par définition de $Z_{a, m_{\Gamma(a)}}$, on a $a c(f)_{\mid X_{a, m_{\Gamma(a)}}}=f_{\gamma}(c)$, donc $X_{a, m_{\Gamma(a)}}$ est de mesure $\left[X_{\gamma}(1), \mu_{m_{\Gamma(a)}}\right] \mathbb{L}^{-s(a)}$.

La contribution de ces termes à la série vaut donc $\sum_{\gamma}\left[X_{\gamma}(1), \mu_{m_{\gamma}}\right] S_{\gamma}(T, \mathbb{L})$. On en déduit la somme totale :

Proposition 2.1.3. Si f est non-dégénérée par rapport à son polyèdre de Newton, on $a$ :

$$
Z_{f}(T)=\mathbb{L}^{-d} \sum_{\gamma}\left(\left[X_{\gamma}(1), \mu_{m_{\gamma}}\right]+\left[X_{\gamma}(0)\right](\mathbb{L}-1) \frac{\mathbb{L}^{-1} T}{1-\mathbb{L}^{-1} T}\right) S_{\gamma}(T, \mathbb{L}) .
$$

Remarque 2.1.4. On peut trouver dans [10] un résultat analogue pour la fonction zêta topologique et dans [9] un énoncé similaire concernant la fonction d'Igusa $p$ adique.

Pour évaluer la limite de cette série, nous utiliserons le lemme suivant.

Lemme 2.1.5. Soit $C$ un cône convexe rationnel polyhédral, $l$ et $l^{\prime}$ deux formes linéaires à valeurs strictement positives sur $C$.

Notons $\mathcal{P}_{C, l}:=\sum_{k \in \stackrel{C}{\circ}} \mathbb{L}^{-l^{\prime}(k)} T^{l(k)}$; alors la limite de la fraction rationnelle $\mathcal{P}_{C, l}$ quand $T \rightarrow \infty$ vaut $(-1)^{\operatorname{dim}(C)}$.

Preuve. Si $C$ est un simplexe dimension $j$ régulier, c'est-à-dire engendré par une partie d'une base $\varepsilon_{1}, \ldots \varepsilon_{j}$, la série considérée vaut $\prod_{i=1}^{j} \frac{T^{l\left(\varepsilon_{i}\right)}}{1-T^{l\left(\varepsilon_{i}\right)}}$ donc tend vers 
$(-1)^{j}$; le cas général s'en déduit en décomposant $C$ en une réunion de simplexes réguliers relativement ouverts disjoints.

On déduit de la proposition 2.1.3 et du lemme 2.1.5 :

Proposition 2.1.6. Si f est non dégénérée par rapport à son polyèdre de Newton on $a$

$$
\mathcal{S}_{f, 0}=\mathbb{L}^{-d} \sum_{\gamma}(-1)^{\operatorname{dim}(\sigma(\gamma))-1}\left(\left[X_{\gamma}(1), \mu_{m_{\gamma}}\right]-\left[X_{\gamma}(0)\right](\mathbb{L}-1)\right)
$$

\section{Espaces d'arcs associés à une courbe plane irréductible}

Notations et conventions. On considère un polynôme $f$ distingué irréductible dans $\mathbf{C}[[x]][y]$ et on suppose que dans le système de coordonnées choisi, la branche de courbe plane à l'origine $C$ définie par $f$ admet une paramétrisation de la forme :

$$
\left\{\sum_{i \geq m} a_{i} u^{i}:=h(u)\right.
$$

avec la condition $\operatorname{pgcd}\left(m,\left\{i \mid a_{i} \neq 0\right\}\right)=1$. Autrement dit, on a dans $\mathbf{C}[[u]][y]$ la factorisation suivante :

$$
f\left(u^{m}, y\right)=\prod_{\zeta^{m}=1}(y-h(\zeta u)) .
$$

On pose $\beta_{1}=\min \left\{i \mid a_{i} \neq 0\right.$ et $\left.m \nmid i\right\}$ et $e_{1}=\left(\beta_{1}, m\right)$; on définit $p_{1}$ et $n_{1}$ par les relations $\beta_{1}=p_{1} e_{1}$ et $m=n_{1} e_{1}$. On définit de même par récurrence : $\forall q \geq 2, \beta_{q}=\min \left\{i \mid a_{i} \neq 0\right.$ et $\left.e_{i-1} \nmid i\right\}, e_{q}=\left(\beta_{q}, e_{q-1}\right)$ et enfin $p_{q}$ et $n_{q}$ par les relations : $\beta_{q}=p_{q} e_{q}$ et $e_{q-1}=n_{q} e_{q}$. On pose $N_{q}=\prod_{i<q} n_{i}$ et $g=\min \left\{q \mid e_{q}=\right.$ $1\}$; remarquons qu'on a la relation $N_{q}=\frac{m}{e_{q}}$.

Les couples $\left(n_{q}, p_{q}\right)_{1 \leq q \leq g}$ sont les paires de Puiseux de $C$. On conviendra également que $\beta_{0}=0, e_{0}=m, N_{0}=1$ ainsi que $\beta_{g+1}=\infty$.

L'ensemble $\Gamma_{C}=\left\{\operatorname{ord}_{u} g(h(u)), g \in \mathcal{O}_{C}\right\}$ est un semi-groupe ([25]). Il admet un système minimal de générateurs noté usuellement $\left\{\bar{\beta}_{0}, \ldots \bar{\beta}_{g}\right\}$. Ces générateurs satisfont les relations

$$
\bar{\beta}_{0}=\beta_{0}, \bar{\beta}_{1}=\beta_{1}, \forall q \geq 2, \bar{\beta}_{q}=n_{q-1} \bar{\beta}_{q-1}+\beta_{q}-\beta_{q-1} .
$$

Rappelons qu'on désigne par $\mathcal{L}\left(\mathbf{A}_{\mathbf{C}}^{2}\right)$ le schéma des arcs tracés dans $\mathbf{A}_{\mathbf{C}}^{2}$; on note $\operatorname{ord}_{t}$ la valuation $t$-adique.

Le but de ce qui suit est de décrire les $\mathbf{G}_{m, \mathbf{C}}$-variétés $X_{n, 1}$ (et l'action de $\mu_{n}$ ) à l'aide du semi-groupe de $f$; pour cela, nous commençons par identifier $\mathcal{L}\left(\mathbf{A}_{\mathbf{C}}^{2}\right)$ à $\mathbf{C}[[t]]^{2}$ via le choix des coordonnées $(x, y)$ puis nous paramétrons les éléments de $\mathbf{C}[[t]]^{2}$ sous une forme normalisée (du type Puiseux). Ceci permet de définir 
une notion de contact (fractionnaire) d'un arc avec $f$; nous classons ensuite ces arcs selon deux paramètres, la valuation en $t$ de leur coordonnée $x$ et leur contact avec $f$.

\subsection{Ecriture normalisée d'un arc et contact avec $f$}

Considérons un arc $\varphi(t):=(x(t), y(t))$ avec $x(t) \neq 0$. Nous pouvons travailler avec une écriture normalisée de $\varphi$ comme le montre le :

Lemme et définition 3.1.1. Il existe $\omega \in \mathbf{C}[[t]]$ et $(X, Y) \in \mathbf{C}[[\omega]]^{2}$ tels que : $\varphi(t)=\left(X(\omega(t)), Y(\omega(t))\right.$ avec $X(\omega)=\omega^{m^{\prime}}$ et $Y(\omega)=\sum_{j \geq 1} b_{j} \omega^{j}$ avec la condition $\operatorname{pgcd}\left(m^{\prime},\left\{j \mid b_{j} \neq 0\right\}\right)=1$.

On appellera une écriture de ce type écriture normalisée de $\varphi$.

Preuve. Désignons par $d$ le pgcd des puissances de $t$ qui figurent dans $x(t)$ et $y(t)$, et posons $x(t)=x^{\prime}\left(t^{d}\right)$ et $y(t)=y^{\prime}\left(t^{d}\right)\left(x^{\prime}\right.$ et $y^{\prime} \in \mathbf{C}[[u]]$ où $u$ désigne une indéterminée) ; alors, si $m^{\prime}$ désigne la valuation de $x^{\prime}$, posons $\tilde{x}(u):=u^{-m^{\prime}} x^{\prime}(u)$ et désignons par $\hat{x}(u)$ une racine $m^{\prime}$-ième de $\tilde{x}(u)$.

L'application $u \mapsto u \hat{x}(u)=: \omega^{\prime}(u)$ est un automorphisme de $\mathbf{C}[[u]]$; on peut donc écrire $y^{\prime}(u):=\sum_{j \geq 1} b_{j} \omega^{\prime}(u)^{j}$.

Si on pose $\omega(t):=\omega^{\prime}\left(t^{d}\right)$, il est clair que le paramétrage $\left(\omega^{m^{\prime}}, \sum_{j \geq 1} b_{j} \omega^{j}\right)$ satisfait aux conditions requises. On peut alors poser la définition suivante :

Définition 3.1.2. Considérons une écriture normalisée de $\varphi$ comme ci-dessus; on appelle ordre de contact de $\varphi$ avec $f$ le maximum des valuations en $x$ des séries fractionnaires $\left(\sum_{j \geq 1} b_{j} \xi^{j} x^{\frac{j}{m^{\prime}}}-\sum_{i \geq m} a_{i} \zeta^{i} x^{\frac{i}{m}}\right)$ (pour $\zeta \in \mu_{m}$ et $\left.\xi \in \mu_{m^{\prime}}\right)$.

Nous avons alors le résultat élémentaire suivant :

Lemme 3.1.3. Soit $r$ un ordre de contact et $q$ l'unique entier tel que $\frac{\beta_{q}}{m}<r \leq$ $\frac{\beta_{q+1}}{m}$ (on a posé $\beta_{g+1}=\infty$ et $\beta_{0}=0$ ); alors, pour tout arc $\varphi$ dont le contact avec $f$ vaut $r, N_{q}$ divise $\operatorname{ord}_{t}(x(\varphi))$.

Preuve. Soit $\varphi$ un arc dont le contact avec $f$ est $r$; puisque le développement fractionnaire normalisé de $\varphi$ coïncide avec l'un des développements de $f$ au moins jusqu'à l' ordre $\frac{\beta_{q}}{m}$ et que cette fraction s'écrit sous forme irréductible $p_{q} / N_{q}$, on a la relation de divisibilité $N_{q} \mid m^{\prime}$ et donc également $N_{q} \mid \operatorname{ord}_{t}(x(\varphi))$.

Ceci nous autorise pour tout entier $l$ à définir $Z_{r, l}$ comme le sous-espace constructible de $\mathcal{L}\left(\mathbf{A}_{\mathbf{C}}^{2}\right)$ des arcs ayant le contact $r$ avec $f$ et tels que ord $\operatorname{or}_{t}(x(t))=l N_{q}$.

Remarquons que tout arc $\varphi$ de $Z_{r, l}$ vérifie la relation

$$
\operatorname{ord}_{t}(f \circ \varphi)=l N_{q}\left(r e_{q}+\frac{\beta_{q}}{m}\left(e_{q-1}-e_{q}\right)+\cdots+\frac{\beta_{1}}{m}\left(e_{0}-e_{1}\right)+\frac{\beta_{0}}{m} e_{0}\right):=\ln (r)
$$


si bien que chaque espace d'arcs $Z_{n}=\left\{\varphi \in \mathcal{L}\left(\mathbf{A}_{\mathbf{C}}^{2}\right), \operatorname{ord}_{t}(f \circ \varphi)=n\right\}$ est une réunion de $Z_{r, l}$.

On pose alors $X_{r, l}=\pi_{\ln (r)}\left(Z_{r, l}\right)$; c'est un sous-ensemble constructible de $X_{\ln (r)}$ naturellement muni d'une structure de $\mathbf{G}_{m, \mathbf{C}}$-variété via la restriction du morphisme $a c(f)$; on notera $X_{r, l, 1}$ pour $\left\{\varphi \in X_{r, l} \mid a c(f)(\varphi)=1\right\}$.

Les résultats suivants décrivent les $\mathbf{G}_{m, \mathbf{C}}$-variétés $X_{r, l}$, selon les valeurs de $r$ et de $l$.

\subsection{Description des espaces d'arcs associés à $f$}

Dans tout ce qui suit, pour tout contact $r \in \mathbf{Q}$ et $l \in \mathbf{N}$, on désigne par $q$ l'unique entier vérifiant $\frac{\beta_{q}}{m}<r \leq \frac{\beta_{q+1}}{m}$; on pose $\nu(r)=r N_{q}$; par définition de $q$, le rationnel $l \nu(r)$ est un entier, sauf si $r=\frac{\beta_{q+1}}{m}$.

Nous commençons par décrire le cas où $q=0$. On notera $\lfloor s\rfloor$ le plus grand entier strictement inférieur à un rationnel $s$.

Lemme 3.2.1. Soit $r$ un ordre de contact vérifiant $0<r<\frac{\beta_{1}}{m}$. On a alors $n(r)=$ rm et $\nu(r)=r$.

i) Si $m r<1$, alors $X_{r, l}$ est isomorphe comme $\mathbf{G}_{m, \mathbf{C}}$-variété à $\mathbf{G}_{m, \mathbf{C}} \times \mathbf{A}_{\mathbf{C}}^{l m r-l r}$ munie du morphisme $c \mapsto c^{m}$; l'action de $\hat{\mu}$ sur $X_{r, l, 1}$ se factorise par l'action de $\mu_{m}$ donnée par $\zeta . c:=\zeta c$.

ii) Si $m r \geq 1$, alors $X_{r, l}$ est isomorphe comme $\mathbf{G}_{m, \mathbf{C}}$-variété à $\left(\mathbf{G}_{m, \mathbf{C}}\right)^{2} \times$ $\mathbf{A}_{\mathbf{C}}^{2 r l m-l-r l}$ munie du morphisme $\left(c, \omega_{l}, \underline{a}\right) \mapsto c^{m}$; l'action de $\hat{\mu}$ sur $X_{r, l, 1}$ se factorise par l'action de $\mu_{m}$ donnée par $\zeta . c:=\zeta c$.

Preuve. Tout arc de $Z_{r, l}$ admet une paramétrisation du type :

$$
\left\{\begin{array}{l}
x(t)=\omega(t) \\
y(t)=\sum_{j \leq r} a_{j} \omega(t)^{j}+c t^{r l}+\sum_{r l<j \leq r l m} b_{j} t^{j}
\end{array}\right.
$$

avec $c \neq 0$.

Dans le cas où $m r<1$, on a $x(t)=0\left[t^{r l m+1}\right]$ et l'espace $X_{\ln (r)}$ est donc paramétré par $\mathbf{G}_{m} \times \mathbf{A}_{\mathbf{C}}^{\ln (r)-l \nu(r)}$, ce qui prouve i).

Si $m r \geq 1, x(t) \neq 0\left[t^{r l m+1}\right]$ et $X_{\ln (r)}$ est donc paramétré par $\left(\mathbf{G}_{m}\right)^{2} \times$ $\mathbf{A}_{\mathbf{C}}^{2 r l m-l-r l}$, ce qui démontre ii).

Traitons maintenant le cas $q \geq 1$.

Lemme 3.2.2. Si $r \neq \frac{\beta_{q+1}}{m}, X_{r, l}$ est isomorphe comme $\mathbf{G}_{m, \mathbf{C}}$-variété à $\left(\mathbf{G}_{m, \mathbf{C}}\right)^{2} \times$ $\mathbf{A}_{\mathbf{C}}^{2 \ln (r)-l \nu(r)-l N_{q}}$ munie du morphisme $\left(c, \omega_{l}, \underline{a}\right) \mapsto c^{e_{q}} \omega_{l}^{\bar{\beta}_{q+1}-\beta_{q+1}}$.

Si $\nu(r)=p / n$ avec $p$ et $n$ premiers entre eux, l'action de $\hat{\mu}$ sur $X_{r, l, 1}$ se factorise par l'action de $\mu_{n n(r)}$ définie par $\zeta .\left(c, \omega_{l}\right):=\left(\zeta^{p} c, \zeta^{n} \omega_{l}\right)$. 
Preuve. Notons $\mathcal{L}_{l, r}\left(\mathbf{A}_{\mathbf{C}}^{2}\right)$ le sous-espace constructible de $\mathcal{L}_{\ln (r)-l N_{q}}\left(\mathbf{A}_{\mathbf{C}}^{2}\right)$ des $\operatorname{arcs}$ tronqués de valuation $l$; on notera $\omega_{l}$ le coefficient de $t^{l}$ dans la série tronquée $\omega$.

Posons $W_{r, l}:=\mathcal{L}_{l, r}\left(\mathbf{A}_{\mathbf{C}}^{2}\right) \times \mathbf{G}_{m, \mathbf{C}} \times \mathbf{A}_{\mathbf{C}}^{\ln (r)-l \nu(r)}$ et définissons un morphisme $\gamma_{r, l}: W_{r, l} \rightarrow X_{r, l}$ par

$$
\gamma_{r, l}(\omega, c, \underline{b}):= \begin{cases}\omega(t)^{N_{q}} & {\left[t^{\ln (r)+1}\right]} \\ \sum_{\frac{j e q}{m} \leq r} a_{j e_{q}} \omega(t)^{j}+c t^{l \nu(r)}+\sum_{l \nu(r)<i \leq l n(r)} b_{i} t^{i}\left[t^{\ln (r)+1}\right]\end{cases}
$$

où on a noté $\underline{b}=\left(b_{l \nu(r)+1}, \ldots, b_{\ln (r)}\right)$.

Cette application a bien un sens; en effet, si $y(t)$ désigne le second terme de l'expression précédente,

$$
\forall \zeta \in \mu_{e_{q}}, \operatorname{ord}_{t}\left(y(t)-h\left(\zeta x(t)^{\frac{1}{m}}\right)\right)=\operatorname{ord}_{t}\left(y(t)-h\left(\zeta \omega(t)^{\frac{1}{e_{q}}}\right)\right)=l \nu(r) .
$$

Ceci prouve que le contact d'un arc de ce type avec $f$ vaut $\frac{l \nu(r)}{N_{q}}=r$.

Quant aux termes correspondant à des racines $m$-ièmes de l'unité non contenues dans $\mu_{e_{q}}$, ils sont de valuation strictement inférieure, ce qui prouve bien que l'image de $\gamma_{r, l}$ est contenue dans $X_{r, l}$.

Montrons que $\gamma_{r, l}$ est surjective. Pour cela considérons $\bar{\varphi} \in X_{r, l}$ et $\varphi \in Z_{r, l}$ tel que $\pi_{\ln (r)}(\bar{\varphi})=\varphi$.

Comme on l'a vu précédemment, $\varphi$ peut se réécrire sous la forme :

$$
\left\{\begin{array}{l}
x(t)=\omega(t)^{m^{\prime}} \\
y(t)=\sum_{j \geq 1} b_{j} \omega(t)^{j}
\end{array}\right.
$$

avec la condition $m^{\prime}=k N_{q}$ pour un $k \in \mathbf{N}$.

L'ordre de contact du développement fractionnaire $\sum_{j \geq 1} b_{j} x^{\frac{j}{m^{\prime}}}$ avec $f$ étant $r$, on en déduit que pour tout $j$ tel que $\frac{j e_{q}}{k m}<r, b_{j}=0$ si $k \nmid j$ et $b_{j}=a_{\frac{j e_{q}}{k}}$ sinon. On a donc, en notant $\omega^{\prime}:=\omega^{k}$, une écriture du type :

$$
\left\{\begin{array}{l}
x(t)=\omega^{\prime}(t)^{N_{q}} \\
y(t)=\sum_{\frac{j e_{q}}{m} \leq r} a_{j e_{q}} \omega^{\prime}(t)^{j}+\sum_{j \geq r m^{\prime}} b_{j} \omega(t)^{j}
\end{array}\right.
$$

avec $a_{r m} \neq b_{r m^{\prime}}$. Ceci montre que $\bar{\varphi}$ s'écrit $\gamma_{r, l}\left(\omega^{\prime}, c, \underline{b^{\prime}}\right)$, avec $\left(0 \neq c=b_{m^{\prime} r}-a_{N_{q} r}\right)$ et donc la surjectivité de $\gamma_{r, l}$.

Considérons désormais la fibre au-dessus de $\bar{\varphi}:=(\bar{x}, \bar{y})$ et conservons les notations précédentes; $\omega$ est solution de l'équation $\omega^{\prime}(t)^{N_{q}} \equiv x(t)\left[t^{\ln (r)}\right]$ et est donc défini à une racine $N_{q}$-ième de l'unité près.

Pour une telle racine $\zeta$, la condition $\bar{\varphi} \in X_{r, l}$ impose le développement de Puiseux de tout relèvement de $\varphi$ jusqu'à l'ordre $r$, donc si $\zeta \omega^{\prime}$ est la première composante d'un autre point de la fibre au-dessus de $\bar{\varphi}$, la condition $\zeta^{j}=1$ est satisfaite 
pour tout $j$ tel que $\frac{j e_{q}}{m}<r$, mais il résulte de la définition de $\beta_{q+1}$ que $\operatorname{pgcd}\left(N_{q},\{j \mid\right.$ $\left.\left.\frac{j e_{q}}{m} \leq r\right\}\right)=1$. Ceci impose $\zeta=1$ et $\omega$ est unique; on obtient ensuite les composantes $c$ et $\underline{b}$ de tout point $(\omega, c, \underline{b})$ de la fibre de $\bar{\varphi}$ en considérant le développement en puissances de $t$ de l' expression $\left(y(t)-\sum_{\frac{j e_{q}}{m} \leq r} a_{j e_{q}} \omega^{\prime}(t)^{j}\right)\left[t^{\ln (r)+1}\right]$ ce qui fournit une unique solution. Il s'ensuit que $\gamma_{r, l}$ est un isomorphisme.

Examinons maintenant $a c(f) \circ \gamma_{r, l}$ :

En raison des propriétés des développements de Puiseux, le terme de valuation minimale dans l'expression $\mathrm{du}\left(y(t)-h\left(\zeta \omega(t)^{\frac{1}{e_{q}}}\right)\right)$ est $c t^{l \nu(r)}$ si $\zeta \in \mu_{e_{q}},\left(\omega_{l} t\right)^{\frac{\beta_{q}}{e_{q}}}$ si $\zeta \in \mu_{e_{q-1}} \backslash \mu_{e_{q}},\left(\omega_{l} t\right)^{\frac{\beta_{q-1}}{e_{q}}}$ si $\zeta \in \mu_{e_{q-2}} \backslash \mu_{e_{q-1}}, \ldots,\left(\omega_{l} t\right)^{\frac{m}{e_{q}}}$ si $\zeta \in \mu_{m} \backslash \mu_{e_{1}} ;$ le coefficient de $t^{\ln (r)}$ dans $f(\bar{x}(t), \bar{y}(t))$ vaut donc $c^{e_{q}} \omega_{l}^{\bar{\beta}_{q+1}-\beta_{q+1}}$.

L'action de $\hat{\mu}$ sur $X_{r, l, 1}$ est induite par l'action de $\mu_{\ln (r)}$ par homothétie sur le paramètre $t$; dans les coordonnées choisies, cette action s'écrit : $\zeta .\left(c, \omega_{l}\right):=$ $\left(\zeta^{l \nu(r)} c, \zeta^{l} \omega_{l}\right)$, ce qui termine la preuve du lemme.

Lemme 3.2.3. Si $r=\frac{\beta_{q+1}}{m}$ et $l \frac{\beta_{q+1}}{e_{q}}=l \frac{p_{q+1}}{n_{q+1}} \notin \mathbf{N}, X_{r, l}$ est isomorphe comme $\mathbf{G}_{m, \mathbf{C}}$-variété à $\mathbf{G}_{m} \times \mathbf{A}_{\mathbf{C}}^{2 \ln (r)-\left\lfloor l p_{q+1} / n_{q+1}\right\rfloor-l N_{q}}$ munie du morphisme $\left(\omega_{l}, \underline{a}\right) \mapsto$ $\omega_{l}^{\bar{\beta}_{q+1}}$. L'action de $\hat{\mu}$ sur $X_{r, l, 1}$ se factorise par l'action de $\mu_{\bar{\beta}_{q+1}}$ définie par $\zeta . \omega_{l}:=$ $\zeta \omega_{l}$

Preuve. Définissons cette fois $W_{r, l}:=\mathcal{L}_{l, r}\left(\mathbf{A}_{\mathbf{C}}^{2}\right) \times \mathbf{A}_{\mathbf{C}}^{\ln (r)-l \nu(r)}$ et $\gamma_{r, l}: W_{r, l} \rightarrow X_{r, l}$ par

$$
\gamma_{r, l}(\omega, c, \underline{b}):=\left\{\begin{array}{lr}
\omega(t)^{N_{q}} & {\left[t^{n(r, l)+1}\right]} \\
\sum_{\frac{j e_{q}}{m}<r} a_{j} \omega(t)^{j}+\sum_{l \nu(r)<i \leq \ln (r)} b_{i} t^{i}\left[t^{n(r, l)+1}\right] .
\end{array}\right.
$$

On montre de même que précédemment que $\gamma_{r, l}$ est un isomorphisme en tenant compte du fait que $l \frac{\beta_{q+1}}{e_{q}}$ n'étant pas entier, le coefficient de $x^{\frac{\beta_{q+1}}{m}}$ dans le développement fractionnaire normalisé de tout relèvement d'un arc tronqué de $X_{r, l}$ est nécessairement nul.

Enfin, il est clair que le coefficient de $t^{\ln (r)}=t^{l \bar{\beta}_{q+1}}$ dans $f(\bar{x}(t), \bar{y}(t))$ est $\omega_{l}^{\bar{\beta}_{q+1}}$, ce qui prouve bien le lemme.

Enfin, on a le résultat suivant :

Lemme 3.2.4. Si $r=\frac{\beta_{q+1}}{m}$ et $l \frac{\beta_{q+1}}{e_{q}}=l \frac{p_{q+1}}{n_{q+1}} \in \mathbf{N}, X_{r, l}$ est isomorphe comme $\mathbf{G}_{m}$-variété à $V_{q+1} \times \mathbf{A}_{\mathbf{C}}^{n(r, l)-\nu(r, l)}$ où

$$
V_{q+1}:=\left\{\left(c, \omega_{l}\right) \in \mathbf{A}_{\mathbf{C}}^{1} \times \mathbf{G}_{m, \mathbf{C}} \mid c^{n_{q+1}}-a_{\beta_{q+1}}^{n_{q+1}} \omega_{l}^{p_{q+1}} \neq 0\right\}
$$

est munie du morphisme $\left(c, \omega_{l}, \underline{a}\right) \mapsto\left(c^{n_{q+1}}-a_{\beta_{q+1}}^{n_{q+1}} \omega_{l}^{p_{q+1}}\right)^{e_{q+1}} \omega_{l}^{\bar{\beta}_{q+1}-\beta_{q+1}}$. 
La variété $X_{r, l, 1}$ est isomorphe à

$$
\left\{(x, y) \in \mathbf{A}_{\mathbf{C}}^{1} \times \mathbf{G}_{m, \mathbf{C}} \mid\left(x^{n_{q}}-y^{\frac{\bar{\beta}_{q}}{e_{q}}}\right)^{e_{q}}=1\right\} .
$$

$L$ 'action de $\hat{\mu}$ sur $X_{r, l, 1}$ se factorise par l'action de $\mu_{n_{q+1} \bar{\beta}_{q+1}}$ définie par $\zeta .(x, y):=$ $\left(\zeta^{\frac{\bar{\beta}_{q+1}}{e_{q+1}}} x, \zeta^{n_{q+1}} y\right)$.

Preuve. On pose

$$
W_{r, l}:=\left\{(\omega, c, \underline{b}) \in \mathcal{L}_{l, r}\left(\mathbf{A}_{\mathbf{C}}^{2}\right) \times \mathbf{G}_{m} \times \mathbf{A}_{\mathbf{C}}^{n(r, l)-\gamma(l, r)} \mid c^{n_{q+1}}-a_{\beta_{q+1}}^{n_{q+1}} \omega_{l}^{p_{q+1}} \neq 0\right\}
$$

et on définit $\gamma_{r, l}: W_{r, l} \rightarrow X_{r, l}$ par

$$
\gamma_{r, l}(\omega, c, \underline{b}):=\left\{\begin{array}{lr}
\omega(t)^{N_{q}} & {\left[t^{\ln (r)}+1\right]} \\
\sum_{\frac{j e q}{m} \leq r} a_{j} \omega(t)^{j}+c t^{l \nu(r)}+\sum_{l \nu(r)<i \leq \ln (r)} b_{i} t^{i}\left[t^{\ln (r)}+1\right] .
\end{array}\right.
$$

Rappelons que $\operatorname{pgcd}\left(\beta_{q+1}, e_{q}\right)=e_{q+1}$ donc l'application $\zeta \mapsto \zeta^{\beta_{q+1}}$ induit un isomorphisme de groupes entre $\left\{\zeta^{\beta_{q+1}} \mid \zeta \in \mu_{e_{q}}\right\}$ et le quotient $\mu_{e_{q}} / \mu_{e_{q+1}}$. En conséquence le produit $\prod_{\zeta \in \mu_{e q}}\left(c-a_{\beta_{q+1}} \omega_{l}^{\frac{\beta_{q+1}}{e_{q}}}\right)$ vaut $\left(c^{n_{q+1}}-a_{\beta_{q+1}}^{n_{q+1}} \omega_{l}^{p_{q+1}}\right)^{e_{q+1}}$, non nul par hypothèse; ceci prouve d'une part que la valuation de chaque facteur $\left(y(t)-h\left(\zeta \omega(t)^{\frac{1}{e_{q}}}\right)\right)$ avec $\zeta \in \mu_{e_{q}}$ est exactement $l \nu(r)$ et que la contribution du produit de tels facteurs au coefficient de $t^{\ln (r)}$ dans $f(\bar{x}(t), \bar{y}(t))$ est $\left(c^{n_{q+1}}-a_{\beta_{q+1}}^{n_{q+1}} \omega_{l}^{p_{q+1}}\right)^{e_{q+1}}$.

La contribution des autres termes (qui ne font pas intervenir $c$ ) se calcule comme dans la preuve du premier lemme et vaut $\omega_{l}^{\bar{\beta}_{q+1}-\beta_{q+1}}$.

Finalement le coefficient de $t^{\ln (r)}$ vaut

$$
\left(c^{n_{q+1}}-a_{\beta_{q+1}}^{n_{q+1}} \omega_{l}^{p_{q+1}}\right)^{e_{q+1}} \omega_{l}^{\bar{\beta}_{q+1}-\beta_{q+1}}
$$

comme annoncé.

On termine la preuve de ce lemme en remarquant que la variété $\{(c, \omega) \in$ $\left.\left.\left.\mathbf{A}_{\mathbf{C}}^{1}\right) \times \mathbf{G}_{m, \mathbf{C}} \mid\left(c^{n_{q+1}}-a_{\beta_{q+1}}^{n_{q+1}} \omega^{p_{q+1}}\right)^{e_{q+1}} \omega^{\bar{\beta}_{q+1}-\beta_{q+1}}=1\right)\right\}$ munie de l'action de $\mu_{n_{q+1} \bar{\beta}_{q+1}}$ définie par $\zeta .\left(c, \omega_{l}\right):=\left(\zeta^{p_{q}} c, \zeta^{n_{q}} \omega_{l}\right)$ est isomorphe à la variété $\{(x, y) \in$ $\left.\mathbf{A}_{\mathbf{C}}^{1} \times \mathbf{G}_{m, \mathbf{C}} \mid\left(x^{n_{q}}-y^{\frac{\bar{\beta}_{q}}{e_{q}}}\right)^{e_{q}}=1\right\}$ munie de l'action $\zeta .(x, y)=\left(\zeta^{\bar{\beta}_{q} / e_{q}} x, \zeta^{n_{q}} y\right)$ (on le vérifie en effectuant le changement de coordonnées sur $\mathbf{A}_{\mathbf{C}}^{1} \times \mathbf{G}_{m, \mathbf{C}}$ donné par : $\left.c=x y^{-\left(\bar{\beta}_{q}-\beta_{q}\right) / n_{q} e_{q}}, \omega=y\right)$. 


\subsection{Calcul de la fibre de Milnor motivique}

Notations. Pour tout $q \in[1 . . g]$, notons $\mathcal{Y}_{q}$ la variété :

$$
\left\{(x, y) \in \mathbf{A}_{\mathbf{C}}^{2} \mid\left(x^{n_{q}}-y^{\frac{\bar{\beta}_{q}}{e_{q}}}\right)^{e_{q}}=1\right\}
$$

munie de l'action de $\mu_{n_{q} \bar{\beta}_{q}}$ donnée par $: \zeta .(x, y):=\left(\zeta^{\frac{\bar{\beta}_{q}}{e_{q}}} x, \zeta^{n_{q}} y\right)$ et $\left[\mathcal{Y}_{q}\right]$ sa classe dans $K_{0}^{\hat{\mu}}\left(\operatorname{Var}_{\mathbf{C}}\right)$.

Rappelons que pour tout entier naturel $n$, on note $\mu_{n}=\operatorname{spec} \mathbf{C}[x] /\left(x^{n}-1\right)$. C'est une variété munie d'une action de $\mu_{n}$ par translation. On notera $\mu_{n}$ sa classe dans $K_{0}^{\hat{\mu}}\left(\operatorname{Var}_{\mathbf{C}}\right)$.

On a alors le résultat :

Proposition 3.3.1. L'égalité suivante est satisfaite dans $\mathcal{M}_{\mathbf{C}}^{\hat{\mu}}$ :

$$
\mathcal{S}_{f, 0}=\sum_{1 \leq q \leq g}\left[\mathcal{Y}_{q}\right]-\mathbb{L} \sum_{1 \leq q \leq g}\left[\mu_{e_{q}}\right]+1
$$

Preuve. Rappelons que pour tout entier $q \in[1 . . g]$ et pour tout ordre de contact $r$, et $l \in \mathbf{N}$, tout arc de $Z_{r, l}$ admet pour valuation

$$
\ln (r):=\operatorname{ord}_{t}(f \circ \varphi)=l N_{q}\left(r e_{q}+\frac{\beta_{q}}{m}\left(e_{q-1}-e_{q}\right)+\cdots+\frac{\beta_{1}}{m}\left(e_{0}-e_{1}\right)+\frac{\beta_{0}}{m} e_{0}\right)
$$

qui vaut $l r m+l\left(n_{q} \bar{\beta}_{q}-\beta_{q}\right)$. Notons que pour $q<g, n_{q} \bar{\beta}_{q}-\beta_{q}=\bar{\beta}_{q+1}-\beta_{q+1}$.

Il résulte des lemmes 3.2.2, 3.2.3 et 3.2.4 que

$$
\begin{aligned}
& Z_{f}(T)=Z_{0}(T)+\sum_{1 \leq q \leq g} Z_{q}(T)+\sum_{1 \leq q \leq g} Z_{q}^{\prime}(T)+\sum_{1 \leq q \leq g} Z^{\prime}{ }_{q}(T) \quad \text { avec : } \\
& Z_{0}(T):=\sum_{0<r<\frac{\beta_{1}}{m}}\left[X_{r, l, 1}\right]\left(\mathbb{L}^{-2} T\right)^{\ln (r)} \\
& Z_{q}^{\prime}(T):=\sum_{l \frac{\beta_{q+1}}{e_{q}} \notin \mathbf{N}}\left[X_{\bar{\beta}_{q+1}, l, 1}^{m}\right]\left(\mathbb{L}^{-2} T\right)^{l \bar{\beta}_{q+1}} \\
& Z_{q}(T):=\sum_{l \frac{\beta_{q+1}}{e_{q}} \in \mathbf{N}}\left[X_{\bar{\beta}_{q+1}}^{m}, l, 1\right]\left(\mathbb{L}^{-2} T\right)^{l \bar{\beta}_{q+1}} \\
& Z^{\prime \prime}(T):= \sum_{\frac{\beta_{q}}{m}<r<\frac{\beta_{q+1}}{m}, l \geq 1}\left[X_{r, l, 1}\right]\left(\mathbb{L}^{-2} T\right)^{\ln (r)} .
\end{aligned}
$$

Calculons les limites de ces sommes:

On utilisera le lemme élémentaire suivant, en notant toujours $\lfloor s\rfloor$ le plus grand entier strictement inférieur à un rationnel $s$. 
Lemme 3.3.2. Soient $p$ et $n$ deux entiers, alors on a

$$
\sum_{l \geq 1} U^{l} V^{\left\lfloor\frac{p l}{n}\right\rfloor}=\frac{1}{1-U^{n} V^{p}} \sum_{1 \leq b \leq n} U^{b} V^{\left\lfloor\frac{p b}{n}\right\rfloor}
$$

en particulier cette série tend vers 0 si $V \rightarrow \infty$ et si $U \rightarrow \infty$ ou reste constant.

Preuve. La première partie résulte d'un calcul; la seconde du fait que $b \geq n$ et $\left\lfloor\frac{p b}{n}\right\rfloor<p$.

Lemme 3.3.3. On a $\lim _{T \rightarrow \infty} Z_{0}(T)=-\left[\mu_{m}\right]$.

Preuve. La somme $Z_{0}(T)$ se décompose en deux termes (correspondant respectivement au ii) et au i) du lemme 3.2.1); plus précisément :

$$
\begin{aligned}
& Z_{0}(T)=\left[\mathcal{X}_{0}\right] \sum_{l \geq 1} \sum_{1 \leq k<\frac{l}{m}}\left(T^{m} \mathbb{L}^{-(m+1)}\right)^{k} \\
& +\left[\mathcal{X}_{0}\right](\mathbb{L}-1) \sum_{l \geq 1} \mathbb{L}^{-l} \sum_{\frac{l}{m} \leq k<l \frac{\beta_{1}}{m}}\left(T^{m} \mathbb{L}^{-1}\right)^{k}, \quad \text { soit encore : } \\
& Z_{0}(T)=\left[\mu_{m}\right] \frac{T^{m} \mathbb{L}^{-(m+1)}}{1-\left(T^{m} \mathbb{L}^{-(m+1)}\right)} \sum_{l \geq 1}\left(1-\left(T^{m} \mathbb{L}^{-(m+1)}\right)^{\left\lfloor\frac{l}{m}\right\rfloor}\right) \\
& +\left[\mu_{m}\right](\mathbb{L}-1) \frac{T^{m} \mathbb{L}^{-1}}{1-\left(T^{m} \mathbb{L}^{-1}\right)} \sum_{l \geq 1} \mathbb{L}^{-l}\left(\left(T^{m} \mathbb{L}^{-1}\right)^{\left\lfloor\frac{l}{m}\right\rfloor}-\left(T^{m} \mathbb{L}^{-1}\right)^{\left\lfloor\frac{\beta_{1}}{m}\right\rfloor}\right) .
\end{aligned}
$$

On déduit du lemme 3.3.2 que $\lim _{T \rightarrow \infty} Z_{0}(T)=-\left[\mu_{m}\right]$.

Evaluons maintenant la limite de $Z_{q}^{\prime}(T)$.

Lemme 3.3.4. On a $\lim _{T \rightarrow \infty} Z_{q}^{\prime}(T)=0$.

Preuve. Rappelons que dans le cas où $l$ vérifie $l \frac{\beta_{q+1}}{e_{q}} \notin \mathbf{N}$, on a d'après le lemme 3.2.3 $X_{r, l, 1}=\mu_{\bar{\beta}_{q+1}} \times \mathbf{A}^{2 l \bar{\beta}_{q+1}-l N_{q}-\left\lfloor l \frac{p_{q+1}}{n_{q+1}}\right\rfloor}$, où $\mathcal{X}_{q+1}^{\prime}$ est la classe de la variété $\mathbf{G}_{m, \mathbf{C}}$ munie de l'action de $\mu_{\beta_{q+1}}$ donnée par $\zeta . \omega=\zeta \omega$.

En particulier $Z_{q}^{\prime}(T)=\left[\mu_{\bar{\beta}_{q+1}}\right] \sum_{l \geq 1, l \frac{\beta_{q+1}}{e_{q}} \notin \mathbf{N}} T^{l \bar{\beta}_{q+1}} \mathbb{L}^{-l N_{q}-\left\lfloor l \frac{p_{q+1}}{n_{q+1}}\right\rfloor}$. Cette somme tend vers zéro par le lemme 3.3.2 et donc $\lim _{T \rightarrow \infty} Z_{q}^{\prime}(T)=0$.

- Nous devons maintenant calculer la limite de $Z_{q}(T)$.

Lemme 3.3.5. On a $\lim _{T \rightarrow \infty} Z_{q}=-\left[\mu_{e_{q+1}}\right]$.

Preuve. Rappelons que si $l \frac{\beta_{q+1}}{e_{q}} \in \mathbf{N}$, on a $X_{r, l, 1}=\mathcal{X}_{q+1} \times \mathbf{A}^{2 l \bar{\beta}_{q+1}-l N_{q}-l \frac{p_{q+1}}{n_{q+1}}}$ d'après le lemme 3.2 .4 , donc $Z_{q}(T)=\left[\mathcal{X}_{q+1}\right] \sum_{l^{\prime} \geq 1} T^{n_{q+1} \bar{\beta}_{q+1}} \mathbb{L}^{-l N_{q+1}-l p_{q+1}}=$ 
$\left[\mathcal{X}_{q+1}\right] \frac{T^{n} q+\bar{\beta}^{\bar{\beta}} q+1 \mathbb{L}^{-N_{q+1}-p_{q+1}}}{1-T^{n_{q+1} \bar{\beta}_{q+1}} \mathbb{L}^{-N_{q+1}-p_{q+1}}}$.

On a donc $\lim _{T \rightarrow \infty} Z_{q}(T)=-\left[\mathcal{X}_{q+1}\right]$.

- Il nous reste enfin à évaluer la limite de $Z{ }{ }_{q}(T)$.

Lemme 3.3.6. On a $\lim _{T \rightarrow \infty} Z{ }^{\prime}{ }_{q}=(\mathbb{L}-1)\left[\mu_{e_{q}}\right]$ si $q \neq g$ et $(\mathbb{L}-1)$ sinon.

Preuve. A l'aide du lemme 3.2.2 on montre que dans le cas où $q \neq g$, on a

$$
Z "{ }_{q}(T)=\left[\mu_{e_{q+1}}\right](\mathbb{L}-1) \sum_{l \geq 1}\left(T^{n_{q} \bar{\beta}_{q}-\beta_{q}} \mathbb{L}^{-N_{q}}\right)^{l} \sum_{k \in \mathbf{N}, \frac{l \beta_{q}}{e_{q}}<k<\frac{l \beta_{q+1}}{e_{q}}}\left(T^{e_{q}} \mathbb{L}^{-1}\right)^{k}
$$

où le coefficient de $\left[\mu_{e_{q+1}}\right](\mathbb{L}-1)$ vaut

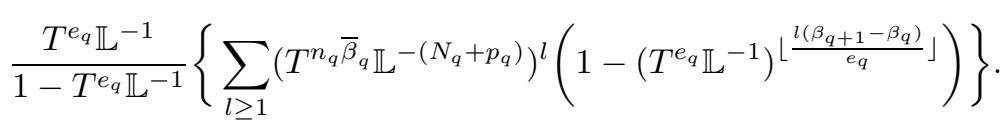

La somme entre accolades tend vers 1 d'après le lemme 3.3.2. Il résulte de ce qui précède que $\lim _{T \rightarrow \infty} Z{ }_{q}(T)=(\mathbb{L}-1)\left[\mu_{e_{q+1}}\right]$ pour $q \neq g$. Dans le cas $q=g$, on remplace la condition $k<\frac{l \beta_{q+1}}{e_{q}}$ par $k<\infty$, et la limite reste la même c'est-à-dire 1 .

Ceci termine la démonstration de la proposition 3.3.1 en notant que $\left[\mathcal{X}_{q+1}\right]=$ $\left[\mathcal{Y}_{q+1}\right]-\left[\mu_{e_{q}}\right]$.

$\diamond$ Exemple : Dans le cas d'une fonction à une seule paire de Puiseux $f(x, y)=$ $y^{\beta_{1}}-x^{m}$, le résultat s'écrit

$$
\mathcal{S}_{f, 0}=\left[\left\{(x, y) \in \mathbf{A}_{\mathbf{C}}^{2} \mid x^{m}-y^{\beta_{1}}=1\right\}\right]-(\mathbb{L}-1) .
$$

\subsection{Application au calcul du spectre}

Nous allons appliquer la proposition 3.3.1 au calcul du spectre de Hodge de $f$. Si l'on écrit

$$
\frac{t^{\frac{e_{q}}{\bar{\beta}_{q}}}-t}{1-t^{\frac{e q}{\bar{\beta}_{q}}}} \cdot \frac{t^{\frac{1}{n_{q}}}-t}{1-t^{\frac{1}{n_{q}}}}=: \sum c_{\alpha} t^{\alpha}
$$

et qu'on pose $\sigma_{q}^{<}(t)\left(\operatorname{resp} . \sigma_{q}^{>}(t)\right):=\sum_{\alpha<1} c_{\alpha} t^{\alpha}\left(\operatorname{resp} . \sum_{\alpha>1} c_{\alpha} t^{\alpha}\right)$, on a le résultat suivant :

Corollaire 3.4.1. Le spectre de Hodge de $f$ vaut :

$$
h \operatorname{sp}(f, 0)=\sum_{1 \leq q \leq g} \frac{1-t}{1-t^{\frac{1}{e_{q}}}}\left\{\sigma_{q}^{<}\left(t^{\frac{1}{e_{q}}}\right)+t^{1-\frac{1}{e_{q}}} \sigma_{q}^{>}\left(t^{\frac{1}{e_{q}}}\right)\right\}
$$

Preuve. On rappelle qu'on peut associer à une variété $\mathcal{X}$, munie d'une bonne action de $\hat{\mu}$, son spectre noté $S p(\mathcal{X})$. C'est la composée de l'application linéaire $h s p$ et 
de la caractéristique de Hodge monodromique $\chi_{h}^{\text {mon }}$ (cf. paragraphe 2). Rappelons que dans notre situation, si on pose $\mathcal{S}_{f, 0}^{\Phi}:=\mathcal{S}_{f, 0}-1$, on a la relation

$$
h s p(f, 0)=-S p\left(\mathcal{S}_{f, 0}^{\Phi}\right) .
$$

On utilisera le lemme élémentaire suivant :

Lemme 3.4.2. Soient $a$ et $b$ deux entiers positifs premiers entre eux. On a :

i) $S p\left(\left\{x \in \mathbf{A}_{\mathbf{C}}^{1} \mid x^{a}=1\right\}\right)=\frac{1-t}{1-t^{\frac{1}{a}}}$

ii) $S p\left(\left\{(x, y) \in \mathbf{A}_{\mathbf{C}}^{2} \mid x^{a}-y^{b}=1\right\}\right)=t-\frac{t^{\frac{1}{a}}-t}{1-t^{\frac{1}{a}}} \cdot \frac{t^{\frac{1}{b}}-t}{1-t^{\frac{1}{b}}}$.

Preuve. Le i) résulte d'un calcul direct.

Posons $Y_{a, b}:=\left\{(x, y) \in \mathbf{G}_{m, \mathbf{C}}^{2} \mid x^{a}-y^{b}=1\right\}$ et remarquons que si $\zeta$ désigne une racine $b$-ième de -1 , l'application $(u, v) \mapsto\left(u^{b}, \zeta v^{a}\right)$ est un isomorphisme entre le quotient de la courbe de Fermat $J_{a b}:=\left\{(u, v) \in \mathbf{G}_{m}^{2} \mid u^{a b}+v^{a b}=1\right\}$ (munie de l'action naturelle de $\mu_{a b}^{2}$ ) par le sous-groupe $\mu_{b} \times \mu_{a}$ et $Y_{a, b}$; cet isomorphisme est équivariant pour l'action induite par l'action diagonale de $\mu_{a b}$ sur $J_{a b}$. Si $(\alpha, \beta)$ est un caractère, on note $I_{\alpha, \beta}$ le sous-espace propre de l'action de $\mu_{a b}^{2}$ sur $H_{c}^{1}\left(J_{a b}\right)$ associé à $(\alpha, \beta)$.

Les sous-espaces propres de $H_{c}^{1}\left(J_{a b} / \mu_{b} \times \mu_{a}\right)$ sont les $I_{\alpha, \beta}$ tels que $(\alpha, \beta)$ soit trivial sur $\mu_{b} \times \mu_{a}$ donc le groupe des caractères de $H_{c}^{1}\left(J_{a b} / \mu_{b} \times \mu_{a}\right)$ s'identifie à $\hat{\mu_{b}} \times \hat{\mu_{a}}$. Si $\alpha \mapsto \tilde{\alpha}$ de $\frac{1}{a} \mathbf{Z} / \mathbf{Z}$ dans $[0,1$ [ désigne la section usuelle, il résulte de [20] lemme 7.1 que le degré pour la filtration de Hodge de $I_{\alpha, \beta}$ ne dépend que de $\tilde{\alpha}+\tilde{\beta}$ sauf dans le cas où $\tilde{\alpha}+\tilde{\beta}=1$; ce cas ne se produit pas ici compte-tenu de la condition $\operatorname{pgcd}(a, b)=1$.

Tous les $I_{\alpha, \beta}$ sont de dimension 1 excepté $I_{0,0}$ dont la dimension est 2 ; par ailleurs, $H_{c}^{2}\left(J_{a b}\right)$ est de dimension 1 , de type de Hodge $(1,1)$ et est associé au caractère trivial. On en déduit que

$$
S p\left(Y_{a, b}\right)=t-\left\{1+\left(1+t^{\frac{1}{a}}+\ldots t^{\frac{a-1}{a}}\right)\left(1+t^{\frac{1}{b}}+\ldots t^{\frac{b-1}{b}}\right)\right\}
$$

d'où

$$
S p\left(\left\{(x, y) \in \mathbf{A}^{2} \mid x^{a}+y^{b}=1\right\}\right)=t-\left(\frac{t^{\frac{1}{a}}-t}{1-t^{\frac{1}{a}}}\right)\left(\frac{t^{\frac{1}{b}}-t}{1-t^{\frac{1}{b}}}\right)
$$

comme annoncé.

Pour finir la preuve du corollaire, nous avons besoin du lemme suivant :

Lemme 3.4.3. Soit $g$ un polynôme en $n$ variables quasi-homogène de degré d (de sorte que pour $c \in \mathbf{G}_{m, \mathbf{C}}$, la variété $\mathcal{Y}:=\left\{x \in \mathbf{A}_{\mathbf{C}}^{n} \mid g(x)=1\right\}$ est munie d'une bonne action de $\left.\mu_{d}\right)$. Soit e un entier positif; alors le spectre de la variété 
$\mathcal{X}$ d'équation $g^{e}=1$ (munie de l'action naturelle de $\mu_{d e}$ ) s'exprime à l'aide du spectre de la variété $\mathcal{Y}$ par la formule:

$$
S p(\mathcal{X})=\sum_{p} t^{p\left(1-\frac{1}{e}\right)} \frac{1-t}{1-t^{\frac{1}{e}}} S p^{(p)}(\mathcal{Y})\left(t^{\frac{1}{e}}\right)
$$

où on a noté $S p(\mathcal{Y}):=\sum c_{\alpha} t^{\alpha}$ et $S p^{(p)}(\mathcal{Y})(t)=\sum_{p \leq \alpha<p+1} c_{\alpha} t^{\alpha}$.

Preuve. La variété $\mathcal{X}$ est la réunion disjointe de $e$ copies de $\mathcal{Y}$ dont chacune est laissée stable par l'action de $T^{e}$; l'action de $T^{e}$ sur une composante s'identifie à l'action de la monodromie naturelle provenant de la quasi-homogénéité de $g$. La décomposition précédente induit une décomposition en somme directe sur les groupes de cohomologie qui est compatible avec la filtration par le poids. Soit donc $H$ une composante de Hodge de poids $p$ de l'un des groupes de cohomologie de $\mathcal{X}$. On a une décomposition de $H$ en sous-espaces propres sous l'action de $\mu_{d e}$ de la forme $H=\bigoplus_{\lambda} H_{\lambda}$ où $\lambda$ décrit $\left\{\exp \left(\frac{2 i k \pi}{d e}\right)\right\}_{0 \leq k \leq d e}$. En regroupant à $\lambda^{e}$ constant, on obtient

$$
S p(\mathcal{X})=\sum_{k=0, \ldots, d-1} t^{\frac{k}{d e}}\left\{\sum_{l=0, \ldots, e-1} t^{\frac{l}{e}} \operatorname{dim} H_{\exp \left(\frac{2 i k \pi}{d e}\right) \exp \left(\frac{2 i l \pi}{e}\right)}\right\} .
$$

L'espace $\bigoplus_{l} H_{\exp \left(\frac{2 i k \pi}{d e}\right) \exp \left(\frac{2 i l \pi}{e}\right)}$ est stable par l'action de $T^{e}$ et s'écrit donc comme somme directe d'espaces propres de cet endomorphisme pour la valeur propre $\exp \left(\frac{2 i k \pi}{d e}\right)$. Or chaque espace de ce type s'identifie au sous-espace propre de la composante de Hodge correspondante d'une composante connexe $\left\{x \in \mathbf{G}_{m, \mathbf{C}}^{d} \mid\right.$ $g(x)=c\}$; en particulier tous ces espaces ont même dimension et cette dimension égale $\operatorname{dim} H(\mathcal{Y})_{\exp \left(\frac{2 i k \pi}{d e}\right)}$. On en déduit que

$$
S p(\mathcal{X})=\sum_{k=0, \ldots, d-1} t^{\frac{k}{d e}} \operatorname{dim} H(\mathcal{Y})_{\exp \left(\frac{2 i k \pi}{d e}\right)}\left(\frac{1-t}{1-t^{\frac{1}{e}}}\right)=t^{-\frac{p}{e}} S p^{(p)}(\mathcal{Y}),
$$

ce qui prouve le lemme.

On déduit de 3.3.1 que $S p\left(\mathcal{S}_{f, 0}^{\Phi}\right)=\sum_{1 \leq q \leq g}-S p\left(\mathcal{Y}_{q}\right)+S p\left(\mu_{e_{q}}\right)$

D'après les lemmes 3.4 .2 et $3.4 .3 \mathrm{ii})$, on a

$S p\left(\mathcal{Y}_{q}\right)=-\left(\frac{1-t}{1-t^{\frac{1}{e_{q}}}}\left(\sigma_{q}^{<}(t)+t^{1-\frac{1}{e_{q}}} \sigma_{q}^{>}(t)\right)\right)+t \frac{1-t}{1-t^{\frac{1}{e_{q}}}}$ et $S p\left(\mu_{e_{q}}\right)=t\left(\frac{1-t}{1-t^{\frac{1}{e_{q}}}}\right)$,

ce qui prouve la formule annoncée.

Donnons deux exemples pour illustrer la formule 3.4.1 :

$\diamond$ Exemple 1 : Si $f$ ne possède qu'une paire de Puiseux $\left(m, \beta_{1}\right)$, on retrouve la formule classique (qui est un cas particulier du calcul du spectre d'une singularité isolée d'hypersurface quasi-homogène)

$$
h \operatorname{sp}(f, 0)=\left(\frac{t^{\frac{1}{m}}-t}{1-t^{\frac{1}{m}}}\right)\left(\frac{t^{\frac{1}{\beta_{1}}}-t}{1-t^{\frac{1}{\beta_{1}}}}\right) .
$$


$\diamond$ Exemple 2: Considérons la courbes à deux paires de Puiseux d'équation $f(x, y):=\left(y^{2}-x^{3}\right)^{2}+y x^{5} ;$ on a $m=4, g=2, e_{1}=2, n_{2}=2$ et $\bar{\beta}_{2}=13$.

On obtient alors $h s p(f, 0)=\sum_{0 \leq i \leq 11} t^{\frac{15+2 i}{26}}+t^{\frac{5}{12}}+t^{\frac{11}{12}}+t^{\frac{13}{12}}+t^{\frac{19}{12}}$. On pourra comparer avec la formule donnée dans [22].

\subsection{Fonction zêta de la monodromie}

On peut déduire de la formule donnée précédemment pour le spectre de Hodge une formule pour la fonction zêta de la monodromie. Remarquons que l'on peut également obtenir cette formule en appliquant le théorème principal de [14].

Corollaire 3.5.1. Soit $\zeta_{f}(t)$ la fonction zêta de la monodromie $T_{f}$ agissant sur la cohomologie de la fibre de Milnor de $f$ et $P_{\Gamma_{C}}(t)$ la série de Poincaré du semigroupe $\Gamma_{C}$. On a la relation : $\left(\zeta_{f}(t)\right)^{-1}=P_{\Gamma_{C}}(t)$.

Preuve. Si $\Lambda\left(T_{f}^{n}\right)$ est le $n$-ième nombre de Lefschetz, on a :

$$
\zeta_{f}(t)=\exp \left(\sum_{n \geq 1} \frac{\Lambda\left(T_{f}^{n}\right)}{n} t^{n}\right)
$$

or d'après [14], $\Lambda\left(T_{f}^{n}\right)$ est égal à la caractéristique d'Euler $\chi\left(X_{n, 1}\right)$.

Calculons ces caractéristiques d'Euler :

Pour tout $n \geq 1$, on a une partition $X_{n, 1}=\coprod_{\ln (r)=n} X_{r, l, 1}$.

Notons $\chi_{r, l}$ pour $\chi\left(X_{r, l, 1}\right)$ et définissons $q$ par l'encadrement $\frac{\beta_{q}}{m}<r \leq \frac{\beta_{q+1}}{m}$; dans le cas où la deuxième inégalité est stricte, on a $\chi_{r, l}=0$ d'après le lemme 3.2.2. Lorsque $r=\frac{\beta_{q+1}}{m}$ on distingue deux cas :

- si $l \frac{\beta_{q+1}}{e_{q}} \notin \mathbf{N}$ (remarquons que ceci équivaut à $n_{q+1} \nmid l$ ), nous sommes dans la situation du lemme 3.2 .3 et $\chi_{r, l}=\bar{\beta}_{q+1}$.

- si $l \frac{\beta_{q+1}}{e_{q}} \in \mathbf{N}$, nous sommes dans la situation du lemme 3.2.4.

$$
\chi_{r, l}=\chi\left(\left\{\left(c, \omega_{l}\right) \mid\left(c^{n_{q+1}}-a_{\beta_{q+1}} \omega_{l}^{p_{q+1}}\right)^{e_{q+1}} \omega_{l}^{\bar{\beta}_{q+1}-\beta_{q+1}}=1\right\}\right)=\bar{\beta}_{q+1}\left(1-n_{q+1}\right)
$$

En effet, l'application $V_{q+1} \rightarrow \mathbf{G}_{m}^{2}$ définie par $\left(c, \omega_{l}\right) \mapsto\left(\left(c^{n_{q+1}}-a_{\beta_{q+1}} \omega_{l}^{p_{q+1}}\right), \omega_{l}\right)$ est un revêtement de degré $n_{q+1}$ ramifié au-dessus de la courbe $\left\{u+a_{\beta_{q+1}} v^{p_{q+1}}=\right.$ 0\} $\subset \mathbf{G}_{m}^{2}$.

Finalement, il vient :

$$
\zeta_{f}(t)=\exp \left(\sum_{l \geq 1} \frac{t^{l m}}{l m}+\sum_{1 \leq q \leq g} \bar{\beta}_{q}\left(\sum_{n_{q} \mid l} \frac{t^{l \bar{\beta}_{q}}}{l \bar{\beta}_{q}}\right)-\bar{\beta}_{q}\left(n_{q}-1\right) \sum_{n_{q} \nmid l} \frac{t^{l \bar{\beta}_{q}}}{l \bar{\beta}_{q}}\right)
$$


soit

$$
\begin{aligned}
\zeta_{f}(t) & =\exp \left(\log \left(1-t^{m}\right)+\sum_{1 \leq q \leq g}\left[\log \left(1-t^{\bar{\beta}_{q}}\right)-\log \left(1-t^{n_{q} \bar{\beta}_{q}}\right)\right]\right) \\
& =\left(1-t^{m}\right) \prod_{1 \leq q \leq g} \frac{\left(1-t^{\bar{\beta}_{q}}\right)}{\left(1-t^{n_{q} \bar{\beta}_{q}}\right)} .
\end{aligned}
$$

Enfin, remarquons que tout élément $n$ de $\Gamma_{C}$ admet une décomposition unique sous la forme $n=\sum_{q=0}^{q=g} \lambda_{q} \bar{\beta}_{q}$ avec $\lambda_{q}$ entiers satisfaisant la condition : $\forall q \in[1 . . g], 0 \leq$ $\lambda_{q} \leq n_{q}-1$ (voir [25]).

La fonction $\left(1-t^{m}\right)^{-1} \prod_{1 \leq q \leq g} \frac{\left(1-t^{n_{q} \bar{\beta}_{q}}\right)}{\left(1-t^{\bar{\beta}} q\right)}$ est donc égale à la série génératrice de $\Gamma_{C}$, ce qui termine la preuve du corollaire.

\section{Fonction d'Igusa motivique associée à plusieurs fonctions}

\subsection{Définition}

Soit $X$ une variété algébrique complexe de dimension $d$ et $p$ fonctions régulières $f_{i}: X \rightarrow \mathbf{A}_{\mathbf{C}}^{1}(i \in\{1, \ldots, p\}) ;$ on pose $X_{0}:=\cap_{1 \leq i \leq p}\left(f_{i}=0\right)$. On notera $f$ l'application $\left(f_{1}, \ldots, f_{p}\right)$.

Chaque fonction $f_{i}$ induit un morphisme $f_{i}: \mathcal{L}(X) \rightarrow \mathcal{L}\left(\mathbf{A}_{\mathbf{C}}^{1}\right) \cong \mathbf{C}[[t]]$ au niveau des espaces d'arcs.

Pour $n \in\left(\mathbf{N}^{\times}\right)^{p}$, il est naturel de considérer le sous-espace d'arcs de $\mathcal{L}(X)$ : $X_{n}:=\left\{\varphi \in \mathcal{L}(X) \mid \forall i \in\{1, \ldots, p\}, \operatorname{ord}_{t} f_{i}(\varphi)=n_{i}\right\}$; c'est un sous-espace constructible de $\mathcal{L}(X)$ naturellement muni d'une structure de $\left(\mathbf{G}_{m, \mathbf{C}}^{p} \times X_{0}\right)$-variété via le morphisme $\left(a c(f), \pi_{0}\right): X_{n} \rightarrow \mathbf{G}_{m, \mathbf{C}}^{p} \times X_{0}$ où $a c(f)$ est l'application qui à un $\operatorname{arc} \varphi \in \mathcal{L}(X)$ associe le $p$-uplet des coefficients de $t^{n_{i}}$ dans $f_{i}(\varphi)(t)$ (pour $i \in\{1, \ldots p\})$. Notons que cet espace est stable à l'ordre $|n|:=\sum_{i=1}^{p} n_{i}$ (voir la section 2$)$; ceci permet de considérer sa mesure $\mu\left(X_{n} / \mathbf{G}_{m, \mathbf{C}}^{p} \times X_{0}\right)$ dans $\mathcal{M}_{\mathbf{G}_{m, \mathbf{C}}^{p} \times X_{0}}$. On pose alors

Définition 4.1.1. La fonction zêta motivique associée à $f$ est l'élément suivant de $\mathcal{M}_{\mathbf{G}_{m, \mathbf{C}}^{p} \times X_{0}}[[T]]$ :

$$
Z_{f}(T):=\sum_{n \in\left(\mathbf{N}^{\times}\right)^{p}} \mu\left(X_{n} / \mathbf{G}_{m, \mathbf{C}}^{p} \times X_{0}\right) T^{n} .
$$

On dispose suivant [20] d'une formule exprimant $Z_{f}(T)$ dans une résolution des singularités des fonctions $f_{1}, \ldots, f_{p}$. On se donne une telle résolution, c'est-à-dire un morphisme propre birationnel $h: Y \rightarrow X$, un diviseur à croisements normaux $E \subset Y$ tel que pour tout $k,\left(f_{k} h\right)^{-1}(0)$ soit supporté par $E$; on note $\left(E_{i}\right)_{i \in J}$ les composantes de $E$ et pour tout $I \subset J$, on définit $E_{I}^{\circ}:=\cap_{i \in I} E_{i} \backslash \cup_{j \notin I} E_{j}$. On note $\lambda^{(i)}$ le $p$-uplet des ordres de $f$ le long du diviseur $E_{i}$ et par $\nu_{i}-1$ l'ordre le long 
de $E_{i}$ de la forme différentielle $h^{*} d x$ où $d x$ est une forme volume sur $X$. On note $U_{i}$ le complémentaire de la section nulle dans le fibré normal à $E_{i}$ et (pour $i \in I$ ), on définit $U_{I}$ comme le produit fibré au-dessus de $E_{I}^{\circ}$ des restrictions $U_{i \mid E_{I}^{\circ}}$; c'est un fibré en tores $|I|$-dimensionnels dont l'espace total est de $\operatorname{dimension} \operatorname{dim} Y$; on note $\pi_{I}$ la projection.

L'application $h \pi_{I}$ fait de $U_{I}$ une variété au dessus de $X_{0}$ et l'application $f h$ : $Y \rightarrow \mathbf{G}_{m, \mathbf{C}}^{p}$ induit de manière naturelle une application de $U_{I}$ dans $\mathbf{G}_{m, \mathbf{C}}^{p}$ que l'on note $\widetilde{f h}$. Il en résulte une structure de $\left(\mathbf{G}_{m, \mathbf{C}}^{p} \times X_{0}\right)$-variété pour $U_{I}$ via $\left(\widetilde{f h}, h \pi_{I}\right)$.

Pour tout $p$-uplet $\nu=\left(\nu_{1}, \ldots, \nu_{p}\right)$, on posera $T^{\nu}=T_{1}^{\nu 1} \ldots T_{p}^{\nu_{p}}$. A l'aide de la formule de changement de variables pour les morphismes birationnels (voir [15] Théorème 4.1.1) et d'un calcul explicite dans la résolution, on montre ([14] pour le cas $p=1$ et $[20])$ que :

\section{Théorème 4.1.2.}

$$
Z_{f}\left(T_{1}, \ldots, T_{p}\right)=\sum_{\emptyset \neq I \subset J}\left[U_{I} / \mathbf{G}_{m, \mathbf{C}}^{p} \times X_{0}\right] \prod_{i \in I} \frac{\mathbb{L}^{-\nu_{i}} T^{\lambda^{i}}}{1-\mathbb{L}^{-\nu_{i}} T^{\lambda^{i}}}
$$

\subsection{Invariants d'Alexander associés à une famille de fonctions}

Ce paragraphe est dévolu au rappel de certaines définitions et propriétés des complexes d'Alexander proches. Nous renvoyons pour toute cette partie à l'article [21]. Dans toute cette section, $X$ désignera une variété complexe lisse de dimension $d$, et $f_{1}, \ldots, f_{p}$ désigneront des fonctions analytiques; on pose $X_{0}:=$ $\cap_{1 \leq i \leq p}\left(f_{i}=0\right)$ et on note $x$ un point de $X_{0}$. On notera $f$ pour $\left(f_{1}, \ldots, f_{p}\right)$; On se donne une résolution des singularités de $f$ pour laquelle on conserve les notations de la section précédente. Sabbah définit dans [21] un foncteur de la catégorie dérivée des faisceaux à cohomologie $\mathbf{C}$-constructible sur $X$ dans la catégorie des complexes à cohomologie $\mathbf{C}\left[\mathbf{Z}^{p}\right]$-constructible sur $X$, qui peut être vu comme une généralisation à plusieurs variables du foncteur cycles proches défini par Deligne (voir la proposition 4.2.1 pour un énoncé précis).

On notera $A$ l'anneau $\mathbf{C}\left[\mathbf{Z}^{p}\right]$. On considère une boule $B$ (suffisamment petite) centrée à l'origine contenue dans $\mathbf{C}^{p}$, on pose $B^{*}:=B \backslash\left(\cup_{1 \leq k \leq p} H_{k}\right)$ ) (où $H_{k}$ désigne le $k$-ième hyperplan de coordonnée) et on note $\widetilde{B^{*}}$ son revêtement universel. On pose également $X^{*}:=X \backslash\left(\cup_{1 \leq i \leq p}\left(f_{i}=0\right)\right)$ et on considère alors le diagramme :

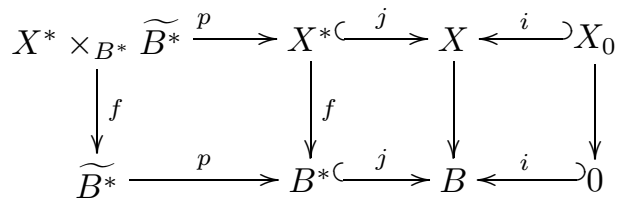

On définit alors, suivant Sabbah, pour tout complexe $\mathcal{F}$ à cohomologie $\mathbf{C}$ - 
constructible sur $X$ le complexe d'Alexander proche

$$
{ }^{A} \psi_{f}(\mathcal{F}):=i^{-1} \mathbf{R} j_{*} \mathbf{R} p_{!} p^{-1} j^{-1} \mathcal{F} \text {. }
$$

C'est un objet de la catégorie $D_{c}^{b}\left(X_{0}, A\right)$.

On dispose du résultat suivant démontré dans [3] concernant les propriétés de ces complexes et le lien avec la théorie des cycles proches associés à une fonction analytique :

Proposition 4.2.1. Si $p=1$ et si $\psi_{f}(\mathcal{F})$ désigne le complexe des cycles proches associé à $f$, on a un quasi-isomorphisme (non canonique) :

$$
{ }^{A} \psi_{f}(\mathcal{F}) \cong \psi_{f}(\mathcal{F})[-1] .
$$

Cycle et fonction zêta d'Alexander. Ce paragraphe est basée sur [21] 2 . Si $Y$ est une variété algébrique complexe ou plus généralement un espace analytique complexe réduit, on dispose pour tout compact $K$ de la notion de fonction (définie au voisinage de $K$ ) analytiquement constructible : une fonction $\alpha$ est analytiquement constructible au voisinage de $K$ si elle est définie sur un voisinage $U$ de $K$ et localement constante sur les strates d'une stratification analytique de $U$. Les exemples typiques sont ceux où $K$ est un point ou bien une composante d'un diviseur exceptionnel dans une résolution des singularités. On note $F_{K}$ le groupe additif engendré par ces fonctions. Si $A$ est un anneau commutatif noethérien unitaire, on note $Z_{K}(A)$ le groupe des cycles sur $A$ à coefficients dans $F_{K}$; un élément de $Z_{K}(A)$ est donc une somme formelle finie $\sum_{\mathfrak{p} \subset A} \alpha_{\mathfrak{p}} V_{\mathfrak{p}}$ où $V_{\mathfrak{p}}$ est la variété associée à l'idéal $\mathfrak{p}$ et $\alpha_{\mathfrak{p}} \in F_{K}$. On dispose de la notion d'image directe pour les fonctions constructibles et donc également pour les cycles : soit $g: X \rightarrow Y$ un morphisme propre, et $K$ un compact de $Y$, l'image directe $g_{*}: Z_{g^{-1}(K)}(A)(X) \rightarrow Z_{K}(A)(Y)$ est définie par

$$
\forall y \in Y, \quad g_{*}(\alpha)(y):=\chi\left(g^{-1}(y), \alpha\right)
$$

où $\chi(, \alpha)$ désigne la caractéristique d'Euler (pour le faisceau constant) pondérée par $\alpha$, c'est-à-dire la fonction constructible $x \mapsto \alpha(x) \chi(x)$.

On note toujours $g_{*}$ l'image directe étendue de manière naturelle aux cycles sur l'anneau $A$.

Si $\mathcal{F}$ est un faisceau $A$-constructible, on appelle support de $\mathcal{F}$ au voisinage de $K$ (ce qu'on note $\operatorname{Supp}_{K}(\mathcal{F})$ ) l'ensemble des idéaux premiers $\mathfrak{p}$ de $A$ tels que $\mathcal{F} \otimes A_{\mathfrak{p}} \neq 0$ au voisinage de $K$; plus généralement, si $\mathcal{F}$ est un objet de $D_{c}^{b}(A)$, on appelle support de $\mathcal{F}$ la réunion des $\operatorname{Supp}_{K} \mathcal{H}^{i}(\mathcal{F})$. Si le support de $\mathcal{F}$ est de dimension $d$, on note $\operatorname{Supp}_{K, d}(\mathcal{F})$ l'ensemble des idéaux du support de $\mathcal{F}$ de cohauteur $d$ dans $A$.

On définit la fonction zêta de $\mathcal{F}$ au voisinage de $K$ comme suit :

Définition 4.2.2. Soit $d$ la dimension du support de $\mathcal{F}$ au voisinage de $K$, alors $\zeta_{K, d}(\mathcal{F})=\sum_{\mathfrak{p} \in \operatorname{Supp}_{K, d}(\mathcal{F})} \chi\left(\mathcal{F}_{\mathfrak{p}}\right) V_{\mathfrak{p}} \in Z_{K}(A)$.

On pose également $\zeta_{K, d}(\mathcal{F})=0$ si $\operatorname{dim} \operatorname{Supp}_{K}(\mathcal{F})<d$. 
Notons le résultat suivant dû à Sabbah, sur le comportement de la fonction zêta par image directe.

Proposition 4.2.3 ([21]). Soit $h: Y \rightarrow X$ propre, lisse et birationnel et $\mathcal{F}$ un complexe à cohomologie A-constructible sur $Y$. Soit $K$ un compact de $X$ tel que Supp $\mathcal{F}$ soit de dimension d au voisinage de $h^{-1}(K)$, alors :

$$
\zeta_{K, d}\left(\mathbf{R} h_{*}(\mathcal{F})\right)=h_{*}\left(\zeta_{f^{-1}(K), d}(\mathcal{F})\right) .
$$

Nous pouvons définir la fonction zêta d'Alexander associée au morphisme $f$ :

Définition 4.2.4. La fonction zêta d'Alexander associée à $f$ au voisinage d'un compact $K$ est le cycle de codimension 1 du complexe ${ }^{A} \psi_{f}(\mathcal{F})$ au voisinage de $K$.

Dans le cas où $\mathcal{F}$ est le faisceau constant sur $X$ et $K=\{x\}$, on notera ${ }^{A} \zeta_{f, x}$ ce cycle. Après avoir fixé des coordonnées sur $\operatorname{Spec} A$, on peut le voir comme une fraction rationnelle en les variables $T_{1}, \ldots, T_{p}$ (définie à un monôme près); nous utiliserons indifféremment les deux notations (additive ou multiplicative) dans la suite pour désigner les fonctions d'Alexander.

Dans [21], Sabbah donne l'expression de la fonction zêta (cf. proposition 4.2.3) en termes d'une résolution des singularités de $f$ qui généralise la formule d'A'Campo [1] sur la fonction zêta de la monodromie :

Proposition 4.2.5 ([21]).

$$
{ }^{A} \zeta_{f, x}\left(T_{1}, \ldots, T_{p}\right)=\prod_{\{i \in J \mid}\left(T^{\lambda^{i}}-1\right)^{\left.-\chi\left(E_{i}\right)=x\right\}}
$$

Lorsque $n=2$, et les $f_{i}$ sont des polynômes irréductibles, cette formule exprime le polynôme d'Alexander de l'entrelac algébrique associé à la singularité de $f_{1} \ldots f_{p}$ (voir [16]).

\subsection{Réalisation d'Alexander}

Nous nous intéressons dans ce paragraphe à des réalisations de $\mathcal{M}_{S}$ dans le cas particulier où $S$ est un tore $\mathbf{G}_{m, \mathbf{C}}^{p}(p \geq 1)$.

Si on se donne une $\mathbf{G}_{m, \mathbf{C}}^{p}$-variété $\mathcal{X} \stackrel{\bar{f}}{\longrightarrow} \mathbf{G}_{m, \mathbf{C}}^{p}$, on dispose d'une notion de cycle d'Alexander sur l'anneau $A$ des polynômes de Laurent à $p$ indéterminées ; en effet, considérons le diagramme : 


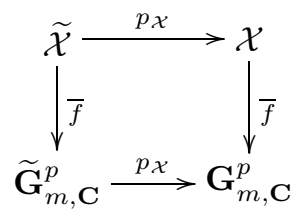

où $\widetilde{\mathbf{G}}_{m, \mathbf{C}}^{p} \stackrel{p_{\mathcal{X}}}{\longrightarrow} \mathbf{G}_{m, \mathbf{C}}^{p}$ désigne le revêtement universel et $\widetilde{\mathcal{X}}$ désigne le produit fibré $\mathcal{X} \times{ }_{\mathbf{G}_{m, \mathbf{C}}^{p}} \tilde{\mathbf{G}}_{m, \mathbf{C}}^{p}$; pour simplifier les notations, on note $p$ pour $p_{\mathcal{X}}$. On définit alors un objet de la catégorie dérivée $D_{c}^{b}(\mathcal{X}, A$-mod) des complexes bornés à cohomologie constructible sur $A$ en posant $\psi_{A}^{*}(\mathcal{X}):=\mathbf{R} p ! p^{-1} \underline{\mathbf{C}}_{\mathcal{X}}$.

Notons que dans cette situation, pour une variété $X_{0}$ et une $\mathbf{G}_{m, \mathbf{C}}^{p} \times X_{0}$-variété $(\bar{f}, \pi): \mathcal{X} \rightarrow \mathbf{G}_{m, \mathbf{C}}^{p} \times X_{0}$, on peut définir un faisceau constructible sur $X_{0}$ en posant $\psi_{A}(\mathcal{X}):=\mathbf{R} \pi ! \psi_{A}^{*}(\mathcal{X}) ;$ on a le résultat suivant :

Lemme 4.3.1. L'application $\mathcal{X} \mapsto \psi_{A}(\mathcal{X})$ s'étend en un morphisme de groupes de $K_{0}\left(\operatorname{Var}_{\mathbf{G}_{m, \mathbf{C}}^{p} \times X_{0}}\right)$ dans $K_{0}\left(D_{c}^{b}\left(X_{0}, A\right)\right)$.

Preuve. Soit $\mathcal{Y}$ un fermé de Zariski de $\mathcal{X}$ et $\mathcal{U}:=\mathcal{X}-\mathcal{Y}$. On définit $p_{\mathcal{X}}: \tilde{\mathcal{X}} \rightarrow \mathcal{X}$, $p_{\mathcal{Y}}: \tilde{\mathcal{Y}} \rightarrow \mathcal{Y}$, et $p_{\mathcal{U}}: \tilde{\mathcal{U}} \rightarrow \mathcal{U}$ comme en début de paragraphe; on notera $p$ pour $p_{\mathcal{X}}$.

On note $i: \mathcal{Y} \hookrightarrow \mathcal{X}(\operatorname{resp} . \tilde{i}: \tilde{\mathcal{Y}} \hookrightarrow \tilde{\mathcal{X}})$ et $j: \mathcal{U} \hookrightarrow \mathcal{X}(\operatorname{resp} . \tilde{j}: \tilde{\mathcal{U}} \hookrightarrow \tilde{\mathcal{X}})$ les inclusions naturelles. En appliquant le foncteur $\mathbf{R} p ! p^{-1}$ au triangle

$$
j ! j^{-1} \underline{\mathbf{C}}_{\mathcal{X}} \rightarrow \underline{\mathbf{C}}_{\mathcal{X}} \rightarrow i_{!} i^{-1} \underline{\mathbf{C}}_{\mathcal{X}}
$$

on obtient un triangle dans $D_{c}^{b}(\mathcal{X}, A)$

$$
\mathbf{R} p ! p^{-1} j_{!} j^{-1} \underline{\mathbf{C}}_{\mathcal{X}} \rightarrow \psi_{A}(\mathcal{X}) \rightarrow \mathbf{R} p ! p^{-1} i_{!} i^{-1} \underline{\mathbf{C}}_{\mathcal{X}} .
$$

D'après le théorème de changement de base, on a

$$
\begin{aligned}
& \mathbf{R} p ! p^{-1} j ! j^{-1} \underline{\mathbf{C}}_{\mathcal{X}}=\mathbf{R} p ! \tilde{j}_{!} p_{\mathcal{U}}^{-1} j^{-1} \underline{\mathbf{C}}_{\mathcal{X}} \\
& =\mathbf{R} p_{\mathcal{U}} ! p_{\mathcal{U}}^{-1} \underline{\mathbf{C}}_{\mathcal{U}} ;
\end{aligned}
$$

en raisonnant de manière analogue pour $\mathcal{Y}$ et en appliquant le foncteur $\mathbf{R} \pi_{0 !}$, on en déduit le triangle

$$
\mathbf{R}\left(\pi_{0} j\right) ! \psi_{A}^{*}(\mathcal{U}) \rightarrow \mathbf{R}\left(\pi_{0}\right) ! \psi_{A}^{*}(\mathcal{X}) \rightarrow \mathbf{R}\left(\pi_{0} i\right) ! \psi_{A}^{*}(\mathcal{Y})
$$

et donc la relation souhaitée dans le groupe de Grothendieck $K_{0}\left(D_{c}^{b}\left(X_{0}, A\right)\right)$.

Si $K$ est un compact de $X_{0}$, on note pour tout $l \in \mathbf{N}$ par $K_{0}\left(D_{c}^{b}\left(X_{0}, A\right)\right)_{K, l}$ le sous-groupe de $K_{0}\left(D_{c}^{b}\left(X_{0}, A\right)\right)$ engendré par les complexes de faisceaux supportés en dimension inférieure à $l$ au voisinage de $K$. On dispose du morphisme $\zeta_{K, p-1}: K_{0}\left(D_{c}^{b}\left(X_{0}, A\right)\right)_{K, p-1} \rightarrow Z_{K}(A)$. En composant, lorsque celà a du sens ce morphisme avec celui défini dans le lemme 4.3.1, on obtient un morphisme de groupes dans $Z_{K}(A)$ que nous noterons ${ }^{A} \zeta_{K}$ et nommerons réalisation d'Alexander. 


\subsection{Fonction d'Igusa associée à une famille de fonctions et invariants d'Alexander}

Nous donnons dans cette section un résultat qui montre que la fonction zêta d'Alexander de la famille $f=\left(f_{1}, \ldots, f_{p}\right)$ peut se déduire de la fonction d'Igusa motivique à plusieurs variables associée à $f$ :

Théorème 4.4.1. Pour tout $\alpha \in\left(\mathbf{N}^{\times}\right)^{p}$ et tout compact $K$ de $X_{0}$, les complexes $\psi_{A}\left(\lim _{T \rightarrow \infty} Z_{f}\left(T^{\alpha}\right)\right)$ et ${ }^{A} \psi_{f}$ sont supportés en codimension strictement positive au voisinage de $K$ et on a l'égalité suivante :

$$
{ }^{A} \zeta_{K}\left(\lim _{T \rightarrow \infty} Z_{f}\left(T^{\alpha}\right)\right)=-{ }^{A} \zeta_{K, f}
$$

Preuve. On déduit du théorème 4.1.2 que pour tout $\alpha \in\left(\mathbf{N}^{\times}\right)^{p}$

$$
\left.\lim _{T \rightarrow \infty} Z_{f}\left(T^{\alpha}\right)\right)=\sum_{\emptyset \neq I \subset J}(-1)^{|I|}\left[U_{I} / \mathbf{G}_{m, \mathbf{C}}^{p}\right] .
$$

Par additivité de la fonction zêta d'Alexander (lemme 4.3.1), on en déduit que que pour tout $\alpha \in\left(\mathbf{N}^{\times}\right)^{p}:{ }^{A} \zeta\left(\lim _{T \rightarrow \infty} Z_{f}\left(T^{\alpha}\right)\right)=\sum_{\emptyset \neq I \subset J}(-1)^{|I|} A_{\zeta_{K}}\left(U_{I} / \mathbf{G}_{m, \mathbf{C}}^{p}\right)$.

En utilisant la proposition 4.2.5, ceci prouve le théorème 4.4.1 modulo le lemme suivant :

Lemme 4.4.2. La relation suivante est vérifiée :

$$
\sum_{\emptyset \neq I \subset J}(-1)^{|I| A} \zeta_{K}\left(U_{I} / \mathbf{G}_{m, \mathbf{C}}^{p}\right)=\sum_{i \in J} \chi\left(E_{i}^{\circ} \cap h^{-1}(K)\right) \mathbb{T}_{\lambda^{i}}
$$

où l'on note $\mathbb{T}_{\lambda}:=\left[\operatorname{Spec}\left(A /\left(T^{\lambda}-1\right)\right)\right]$ pour $\lambda \in\left(\mathbf{N}^{\times}\right)^{p}$.

Preuve. En appliquant la proposition 4.2.3, nous sommes amenés à calculer le cycle d'Alexander de la $\mathbf{G}_{m, \mathbf{C}}^{p} \times E_{I}^{\circ}$-variété $\left(\tilde{f} h, \pi_{I}\right): U_{I} \rightarrow \mathbf{G}_{m, \mathbf{C}}^{p} \times E_{I}^{\circ}$.

Par additivité de la fonction zêta d'Alexander (lemme 4.3.1) nous pouvons nous contenter de travailler sur un ouvert affine $V$ de $Y$ tel que $U_{I}$ est trivial au-dessus de $V \cap E_{I}^{\circ}$. Nous sommes donc ramenés à calculer la fonction zêta d'Alexander dans le cas où $\tilde{f h}$ est un morphisme de $\left(E_{I}^{\circ} \cap V\right) \times \mathbf{G}_{m, \mathbf{C}}^{k}(k:=|I|)$ dans $\mathbf{G}_{m, \mathbf{C}}^{p}$ donné par $\left(x, y_{1}, \ldots, y_{k}\right) \mapsto\left(u_{1} \prod_{i=1}^{k} y_{i}^{\lambda_{1}^{i}}, \ldots, u_{p} \prod_{i=1}^{k} y_{i}^{\lambda_{p}^{i}}\right)$, les $u_{j}$ désignant des unités.

On note $r$ le rang du sous-module de $\mathbf{Z}^{p}$ engendré par $\lambda^{1}, \ldots, \lambda^{p}$.

Si $r<k$, l'application $\left(E_{I}^{\circ} \cap V\right) \times \mathbf{G}_{m, \mathbf{C}}^{k} \rightarrow \mathbf{G}_{m, \mathbf{C}}^{p}$ se factorise par la projection sur un sous-tore $\mathbf{G}_{m, \mathbf{C}}^{r}$ donc $\psi_{A}^{*}\left(\left(E_{I}^{\circ} \cap V\right) \times \mathbf{G}_{m, \mathbf{C}}^{k}\right) \cong \psi_{A}^{*}\left(\left(E_{I}^{\circ} \cap V\right) \times \mathbf{G}_{m, \mathbf{C}}^{r}\right) \otimes \underline{A}_{\mathbf{G}_{m, \mathbf{C}}^{k-r}}$. Comme $k>r$, pour tout idéal $\mathfrak{p}$ de $A$ on a $\chi\left(\left(\psi_{A}^{*}\left(\left(E_{I}^{\circ} \cap V\right) \times \mathbf{G}_{m, \mathbf{C}}^{k}\right)\right)_{\mathfrak{p}}\right)=0$ et donc le cycle d'Alexander de ce complexe est nul.

Par ailleurs, il résulte de [21] que si $f: \mathcal{X} \rightarrow \mathbf{G}_{m, \mathbf{C}}^{a}$ est un morphisme et $\gamma: \mathbf{G}_{m, \mathbf{C}}^{a} \rightarrow \mathbf{G}_{m, \mathbf{C}}^{b}$ un morphisme de groupes qui fait de $\mathcal{X}$ une variété au-dessus 
de $\mathbf{G}_{m, \mathbf{C}}^{b}$, alors on a

$$
\psi_{A}^{*}\left(\mathcal{X} / \mathbf{G}_{m, \mathbf{C}}^{b}\right) \cong \psi_{A}^{*}\left(\mathcal{X} / \mathbf{G}_{m, \mathbf{C}}^{a}\right) \otimes_{\mathbf{C}\left[\mathbf{Z}^{a}\right]} \mathbf{C}\left[\mathbf{Z}^{b}\right]
$$

où $\mathbf{C}\left[\mathbf{Z}^{b}\right]$ est vu comme $\mathbf{C}\left[\mathbf{Z}^{a}\right]$-module via le morphisme induit par $\gamma$ sur les groupes fondamentaux.

Lorsque $k=r$, on peut par des isomorphismes de tores convenables à la source et au but se ramener au cas du morphisme

$$
\left(x, y_{1}, \ldots, y_{r}\right) \mapsto\left(u_{1}(x) y_{1}^{a_{1}}, \ldots, u_{r}(x) y_{r}^{a_{r}}, u_{r+1}(x), \ldots, u_{p}(x)\right)
$$

(où les $u_{j}$ sont des unités).

On en déduit que l'annulateur du faisceau d'Alexander correspondant est $S_{1}^{a_{1}}-$ $1, \ldots, S_{r}^{a_{r}}-1$ (où les $S_{i}$ sont des coordonnées convenablement choisies sur $A$ ); en particulier, son support est de codimension $r$ donc son cycle de codimension un est nul dès que $r \geq 2$.

Il reste à traiter le cas $r=1$. En appliquant $(*)$ pour le morphisme de groupes $\mathbf{G}_{m, \mathbf{C}} \rightarrow \mathbf{G}_{m, \mathbf{C}}^{p}$ donné par $y \mapsto\left(y^{a_{1}}, \ldots, y^{a_{r}}\right)$, on obtient $\psi_{A}\left(\left(E_{I}^{\circ} \cap V\right) \times \mathbf{G}_{m, \mathbf{C}}\right) \cong$ $\mathbf{C}\left[T_{1}^{1}, T_{1}^{-1}, \ldots T_{p}^{1}, T_{p}^{-1}\right] /\left(T_{1}^{a_{1}} \ldots T_{p}^{a_{p}}-1\right) \otimes_{A} \underline{A}_{\left(E_{I}^{\circ} \cap V\right)}$; la fonction zêta d'Alexander est donc égale à $\chi\left(E_{I}^{\circ} \cap V\right) \mathbb{T}_{a}$ comme annoncé.

\section{Application aux courbes planes réductibles}

Arbre des contacts ; notations. On se donne dans cette section $p$ polynômes de Weierstrass irréductibles dans $\mathbf{C}[[x]][y]$ notés $f_{1}, \ldots, f_{p}$ et on cherche à exprimer la fonction zêta d'Alexander de l'entrelac défini par $f_{1}, \ldots, f_{p}$ en fonction des données de Puiseux de chaque branche et des contacts entre ces branches. On choisit des coordonnées $x$ et $y$ telles que la fonction $x$ soit transverse à chacune des $f_{i}$, ce qui est toujours possible. Pour chaque $f_{i}$, on définit comme au paragraphe 3 des suites d'entiers $m^{(i)}, \beta_{q}^{(i)}, e_{q}^{(i)}, n_{q}^{(i)}, N_{q}^{(i)}$ et $\bar{\beta}_{q}^{(i)}$ (pour $q \in\left\{1, \ldots, g^{(i)}\right\}$ ) ; notons qu'avec notre choix de coordonnées on a nécessairement $\forall i \in\{1, \ldots p\} \beta_{1}^{(i)} \geq m^{(i)}$; on pose par ailleurs $\tilde{\beta}_{q}^{(i)}:=\bar{\beta}_{q+1}^{(i)}-\beta_{q+1}^{(i)}$ et on note $C_{i, j}$ la multiplicité d'intersection à l'origine des branches $f_{i}$ et $f_{j}$. On note également $r_{i, j}$ le contact de $f_{i}$ et de $f_{j}$, c'est-à-dire le contact d'une paramétrisation de $f_{i}^{-1}(\{0\})$ avec la fonction $f_{j}$ au sens de la section 2 de sorte qu'on a la relation

$$
C_{i, j}=m^{(j)}\left(r_{i, j}+\frac{\beta_{q_{\left(r_{i, j}\right)}^{(i)}}^{m}}{m}\left(e_{q\left(r_{i, j}\right)-1}^{(i)}-e_{q\left(r_{i, j}\right)}^{(i)}\right)+\cdots+\frac{\beta_{1}^{(i)}}{m}\left(e_{0}^{(i)}-e_{1}^{(i)}\right)+\frac{\beta_{0}^{(i)}}{m} e_{0}^{(i)}\right) .
$$

Pour tout $i \in\{1, \ldots, p\}$, on note $\sum_{s \in \mathbf{Q}} a_{s}^{(i)} x^{s}$ l'un des développements fractionnaires associé à $f_{i}$. On représente chaque développement fractionnaire correspondant aux différentes branches $f_{i}$ par un arbre où l'on indique les exposants (rationnels) et les coefficients correspondants. Pour tout rationnel $r$, l'ensemble des tronqués à l'ordre $r$ des développements de Puiseux associés à ces arbres 
est muni de l'action d'un groupe de racines de l'unité (correspondant au pgcd des dénominateurs des exposants fractionnaires des développements tronqués envisagés). On identifie dans la réunion disjointe de tous les arbres considérés les sous-arbres correspondant à des développements tronqués contenus dans la même orbite pour cette action; on appelle arbre des contacts le diagramme ainsi obtenu.

Sur cet arbre sont donc indiqués tous les points correspondant à un exposant fractionnaire qui apparait dans au moins l'un des développements de Puiseux, ainsi que, pour chaque exposant $r$, le coefficient de $x^{r}$ dans le développement envisagé. On appellera sommet la classe d'un point de ramification du diagramme, branche tout segment joignant deux sommets, et feuille toute demi-droite dont un sommet est un point d'altitude maximale de l'arbre. On notera $\mathcal{B}$ l'ensemble des branches et des feuilles. Chaque sommet $S$ de l'arbre des contacts correspond de manière naturelle à l'orbite d'un développement tronqué $\sum_{s<h(S)} a_{s}^{(i)} x^{s}(h(S) \in \mathbf{Q})$; on note $I_{S}$ l'ensemble des $i \in\{1, \ldots, p\}$ tels que le tronqué à l'ordre $h(S)$ de l'un des développements de Puiseux associé à $f_{i}$ coïncide avec ce développement fractionnaire, et $J_{S}$ son complémentaire. On appellera une expression $\sum_{s<h(S)} a_{s}^{(i)} x^{s}$ (qui est indépendante de $\left.i \in I_{S}\right)$ développement fractionnaire associé au point $S$.

Notons que l'entier $q(S):=\min \left\{q^{\prime} \mid \frac{\beta_{q^{\prime}}^{(i)}}{m^{(i)}} \leq h(S)\right\}$ est indépendant de $i \in I_{S}$ (nous le noterons simplement $q$ lorsque le contexte sera clair). Si $j \in J_{S}$, on note $C_{S}(j)$ le nombre $\frac{C_{i, j}}{m^{(i)}}$ (qui ne dépend pas de $i \in I_{S}$ ); par ailleurs, on divise $I_{S}$ en deux sous-ensembles $I_{S}^{\prime}$ et $I^{\prime \prime}{ }_{S}$; le premier désigne $\left\{i \in I_{s} \mid h(S)\right.$ n'est pas un exposant de Puiseux de $\left.f_{i}\right\}$ et $I "{ }_{S}$ est son complémentaire dans $I_{S}$.

On pose $k^{\prime}(S):=\left|I_{S}^{\prime}\right|$ et $k^{\prime \prime}(S):=\left|I^{\prime \prime}\right|$.

On distingue dans ce diagramme deux types de sommets : les sommets du type 1 sont ceux pour lesquels $I_{S}^{\prime \prime}=\emptyset$; on note $I_{1}$ l'ensemble de ces sommets les autres sommets étant appelés sommets du type 2 (on notera $I_{2}$ l'ensemble des sommets du type 2).

\subsection{Description des espaces d'arcs associés à plusieurs fonctions}

Rappelons que si un rationnel $r$ vérifie l'encadrement $\frac{\beta_{q}^{(i)}}{m}<r \leq \frac{\beta_{q+1}^{(i)}}{m}$, on pose :

$n^{(i)}(r):=N_{q}^{(i)}\left(r e_{q}^{(i)}+\frac{\beta_{q}^{(i)}}{m}\left(e_{q-1}^{(i)}-e_{q}^{(i)}\right)+\cdots+\frac{\beta_{1}^{(i)}}{m}\left(e_{0}^{(i)}-e_{1}^{(i)}\right)+\frac{\beta_{0}^{(i)}}{m} e_{0}^{(i)}\right)$.

On rappelle que l'ordre de $f_{i}$ le long d'un arc ayant le contact $r$ avec $f_{i}$ et dont la coordonnée $x$ a pour valuation $l N_{q}^{(i)}$ est égal à $\ln ^{(i)}(r)$.

Nous nous donnons désormais $\varphi \in \mathcal{L}\left(\mathbf{A}_{\mathbf{C}}^{2}\right)$ et nous posons la

Définition 5.1.1. On appelle contact de $\varphi$ avec $f:=\left(f_{1}, \ldots, f_{p}\right)$ le maximum des contacts de $\varphi$ avec les $f_{i}$ (au sens du lemme 3.1.2).

A tout arc de contact $r$ correspond à la classe d'un développement tronqué qui 
aboutit en un sommet ou en un point d'une branche noté $S$ et vérifiant $h(S)=r$.

Pour chaque sommet $S$, on définit $Z_{S, l}$ comme le sous-espace constructible des arcs dont le contact avec $f$ est réalisé pour un développement tronqué aboutissant en $S$ et dont la valuation en $x$ vaut $l N_{q(S)}$.

On pose $n(r):=\sum_{i=1}^{p} n^{(i)}(r)$ et on considère $X_{S, l}:=\pi_{\ln (r)}\left(Z_{S, l}\right)$ muni de sa structure naturelle de $\mathbf{G}_{m, \mathbf{C}^{-}}^{p}$ variété via le morphisme $a c(f)$. Le but de ce paragraphe est de décrire les $\mathbf{G}_{m, \mathbf{C}^{p}}^{p}$-variétés $X_{S, l}$ en fonction de l'arbre des contacts.

- Soit $S_{0}$ le sommet d'altitude minimale $h\left(S_{0}\right)$ (qui est strictement positive sauf si $p=1$ et $f$ lisse, auquel cas nous conviendrons que $h\left(S_{0}\right)=\infty$. Notons $m:=N_{q\left(S_{0}\right)}$.

Nous commençons par étudier le cas des arcs dont le contact avec $f$ est strictement inférieur à $h\left(S_{0}\right)$. On a le résultat suivant :

Lemme 5.1.2. Soit $r$ un ordre de contact vérifiant $0<r<h\left(S_{0}\right)$ :

i) Si $m r<1$, alors $X_{S, l}$ est isomorphe comme $\mathbf{G}_{m, \mathbf{C}}^{p}$-variété à $\mathbf{G}_{m, \mathbf{C}} \times \mathbf{A}_{\mathbf{C}}^{\ln (r)-l r}$ munie du morphisme $c \mapsto\left(c^{m^{(i)}}\right)_{1 \leq i \leq p}$.

ii) Si $m r \geq 1$, alors $X_{S, l}$ est isomorphe comme $\mathbf{G}_{m, \mathbf{C}}^{p}$-variété à $\left(\mathbf{G}_{m, \mathbf{C}}\right)^{2} \times$ $\mathbf{A}_{\mathbf{C}}^{2 \ln (r)-l-r l}$ munie du morphisme $\left(c, \omega_{l}, \underline{a}\right) \mapsto\left(c^{m^{(i)}}\right)_{1 \leq i \leq p}$.

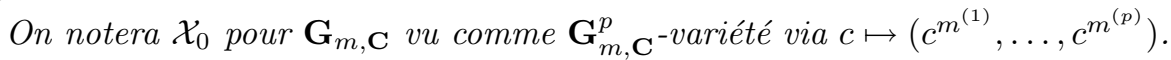

Preuve. On peut paramétrer un arc de $Z_{S, l}$ sous la forme :

$$
\left\{\begin{array}{l}
x(t)=\omega(t) \\
y(t)=\sum_{s<r} a_{s} \omega(t)^{s}+c t^{r l}+\sum_{r l<j \leq r l m} t^{j} .
\end{array}\right.
$$

Dans le cas où $m r<1$, on a $x(t)=0\left[t^{\ln (r)+1}\right]$ et l'espace $X_{\ln (r)}$ est donc paramétré par $\left(\mathbf{G}_{m, \mathbf{C}}\right) \times \mathbf{A}_{\mathbf{C}}^{\ln (r)-l}$, ce qui prouve i).

Si $r \geq 1, \omega(t) \neq 0\left[t^{\ln (r)+1}\right]$ et $X_{\ln (r)}$ est paramétré par $\left(\mathbf{G}_{m, \mathbf{C}}\right)^{2} \times \mathbf{A}_{\mathbf{C}}^{2 \ln (r)-l-r l}$, ce qui démontre ii).

- Etudions ensuite le cas où $S$ est un sommet du type 1 ou bien une branche de l'arbre des contacts, définissons $\mathcal{X}_{S}$ comme la $\mathbf{G}_{m, \mathbf{C}}^{p}$-variété

$$
\mathbf{A}_{\mathbf{C}}^{1} \backslash\left\{a^{(i)}\right\}_{i \in I_{S}} \times \mathbf{G}_{m, \mathbf{C}} \stackrel{\bar{f}_{S}}{\longrightarrow} \mathbf{G}_{m, \mathbf{C}}^{p}
$$

où $\bar{f}_{S}(c, \omega)$ est défini par les identités :

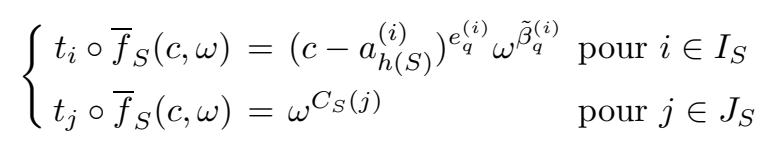

(on a noté $t_{1}, \ldots, t_{p}$ les coordonnées dans $\left(\mathbf{G}_{m, \mathbf{C}}\right)^{p}$ et $c$ et $\omega$ les coordonnées à la source). 
Dans le cas où $S$ est une branche $B$, on notera $\mathcal{X}_{B}$ cette même classe $\left(I_{S}\right.$ est réduit à un point dans ce cas).

On a alors l'énoncé suivant :

Lemme 5.1.3. Si $S$ est un sommet du type 1 ou bien une branche du diagramme des contacts, $X_{S, l}$ est isomorphe comme $\mathbf{G}_{m, \mathbf{C}}^{p}$-variété à $\mathcal{X}_{S} \times \mathbf{A}^{2 \ln (r)-l \nu(r)-l N_{q}}$.

Preuve. Remarquons que puisque les développements des $f_{j}$ (pour $j \in I_{S}$ ) coïncident jusqu'à l'ordre $r$ (non inclus), il existe un entier $q$ tel que $r$ satisfait l'encadrement $\frac{\beta_{q}^{(j)}}{m}<r \leq \frac{\beta_{q+1}^{(j)}}{m}$. Les conditions de coïncidence imposent que le terme de gauche de cet encadrement est indépendant de $j \in I_{S}$. Nous le noterons simplement $\frac{\beta_{q}}{m}$. On notera de même $N_{q}$ pour $N_{q}^{(j)}$ et $a_{s}$ pour $a_{s}^{(j)}$.

Il résulte de l'étude effectuée pour les courbes irréductibles que $\varphi$ admet une paramétrisation de la forme :

$$
\left\{\begin{array}{l}
x(t)=\omega(t)^{N_{q}} \\
y(t)=\sum_{s<r} a_{s} \omega(t)^{s N_{q}}+c t^{\nu}+\sum_{s>\nu} b_{s} t^{s}
\end{array}\right.
$$

où $c \in \mathbf{A}_{\mathbf{C}}^{1} \backslash\left\{a^{(i)}\right\}_{i \in I_{S}}$.

Autrement dit, pour tout $l \in \mathbf{N}, X_{r, l}$ s'identifie au produit d'un espace affine $\operatorname{par} \mathbf{A}_{\mathbf{C}}^{1} \backslash\left\{a^{(i)}\right\}_{i \in I_{S}} \times \mathbf{G}_{m, \mathbf{C}}$.

On déduit des résultats obtenus pour une fonction que le $p$-uplet des exposants de $f$ le long de $\varphi$ vaut $n:=\left(n_{1}, \ldots, n_{p}\right)$ avec $n_{i}=\ln ^{(i)}(r)$ pour $i \in I_{S}$, et $n_{j}=l C_{S}(j)$ : il résulte en effet de la définition de $J_{S}$ que l'ordre de la fonction $f_{j}\left(j \in J_{S}\right)$ d'un arc aboutissant en $S$ s'obtient en évaluant l'ordre de $f_{j}$ sur l'un des développements de Puiseux d'une fonction $f_{i}$ (pour $i$ quelconque dans $I_{S}$ ). On déduit également du cas d'une seule fonction l'égalité : $a c(f)(\varphi)=\bar{f}_{S}(c, \omega)$.

- Lorsque $S$ est un sommet du type 2, nous avons $I_{S}^{\prime \prime}=\left\{j \mid r=\frac{\beta_{q+1}^{(j)}}{m^{(j)}}\right\} \neq \emptyset$. Nous devons alors envisager deux cas :

- Soit la condition $l \frac{\beta_{q+1}^{(i)}}{e_{q}^{(i)}} \notin \mathbf{N}$ est vérifiée (notons que compte-tenu des hypothèses, cette condition est indépendante de $j \in I$ ). On notera dans ce cas $\mathcal{X}^{\prime}{ }_{S}$ la $\mathbf{G}_{m, \mathbf{C}}^{p}$-variété $\mathbf{G}_{m, \mathbf{C}} \stackrel{\bar{f}_{S}^{\prime}}{\longrightarrow} \mathbf{G}_{m, \mathbf{C}}^{p}$ où $\bar{f}_{S}^{\prime}$ est défini par les relations :

$$
\begin{cases}t_{i} \circ \overline{f^{\prime}}{ }_{S}(c, \omega)=\omega^{\tilde{\beta}_{q}^{(i)}} & \text { pour } i \in I_{S} \\ t_{j} \circ \overline{f^{\prime}}{ }_{S}(c, \omega)=\omega^{C_{S}(j)} & \text { pour } j \in J_{S} .\end{cases}
$$

Lemme 5.1.4. Si $S$ est du type 2 et $l \frac{\beta_{q+1}^{(i)}}{e_{q}^{(i)}} \notin \mathbf{N}, X_{S, l}$ est isomorphe comme $\mathbf{G}_{m, \mathbf{C}^{-}}^{p}$ variété à $\mathcal{X}_{S}^{\prime} \times \mathbf{A}_{\mathbf{C}}^{2 \ln (r)-\left\lfloor l \beta_{q+1}^{(i)} / e_{q}^{(i)}\right\rfloor-l N_{q}}$. 
Preuve. Il résulte de l'étude effectuée pour une seule fonction que $\varphi$ admet une paramétrisation du type suivant :

$$
\left\{\begin{array}{l}
x(t)=\omega(t)^{N_{q}} \\
y(t)=\sum_{s<r} a_{s} \omega(t)^{s N_{q}}+\sum_{\mu>\nu} b_{s} t^{s}
\end{array}\right.
$$

où $\nu:=\operatorname{lr} N_{q}$ (c'est un entier sous nos hypothèses).

En effet, dans tous les termes correspondant à des valeurs de $j$ contenues dans $J_{S}$, on a $r<\frac{\beta_{q+1}^{(j)}}{m^{(j)}}$, donc la contribution du terme en $x^{r}$ au développement fractionnaire de $f_{j}(x, \bar{y}(x))(\bar{y}(x)$ désigne un développement fractionnaire associé à $\varphi$ normalisé comme dans le lemme 3.1.1) est apportée par le terme en $t^{\nu}$; comme ce terme (qui vaut $l \frac{\beta_{q+1}^{(i)}}{e_{q}^{(i)}}$ ) n'est pas entier par hypothèse, son coefficient $c$ est nécessairement nul, ce qui justifie le paramétrage précédent.

Dans cette situation, le $p$-uplet des exposants de $f$ le long de $\varphi$ vaut $n:=$ $\left(n_{1}, \ldots, n_{p}\right)$ avec $n_{i}=l \bar{\beta}_{q+1}^{(i)}$ si $i \in I_{S}$ et $n_{j}=\ln ^{(j)}(r)$ si $j \in J_{S}$ et l'espace $X_{r, l}$ s'identifie au produit d'un espace affine par $\mathbf{C}^{*}$ sur lequel on note $\omega_{l}$ une coordonnée. On a alors l'égalité $a c(f)(\varphi)=\bar{f}_{S}^{\prime}(c, \omega)$.

- Soit la condition $l \frac{\beta_{q+1}^{(j)}}{e_{q}^{(j)}} \in \mathbf{N}$ est vérifiée. On notera $\mathcal{X}{ }_{S}$ la $\mathbf{G}_{m, \mathbf{C}}^{p}$-variété : $V_{S} \stackrel{\overline{f^{\prime}}}{\longrightarrow} \mathbf{G}_{m, \mathbf{C}}^{p}$, où $\overline{f_{S}^{\prime \prime}}(c, \omega)$ est défini par les relations suivantes :

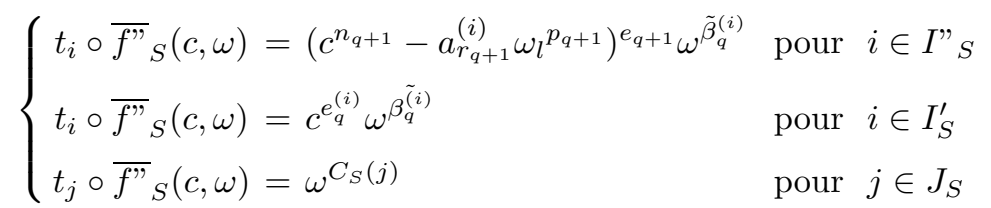

l'ensemble $I_{S}^{\prime}$ étant éventuellement vide.

Lemme 5.1.5. Si $S$ est du type 2 et $l \frac{\beta_{q+1}^{(i)}}{e_{q}^{(i)}} \in \mathbf{N}, X_{S, l}$ est isomorphe comme $\mathbf{G}_{m, \mathbf{C}^{-}}^{p}$ variété à $\mathcal{X}{ }_{S} \times \mathbf{A}_{\mathbf{C}}^{2 \ln (r)-l \nu(r)-l N_{q}}$.

Preuve. Dans cette situation, $\varphi$ admet un paramétrage de la forme :

$$
\left\{\begin{array}{l}
x(t)=\omega(t)^{N_{q}} \\
y(t)=\sum_{s<r} a_{s} \omega(t)^{s N_{q}}+c t^{\nu}+\sum_{s>\nu} b_{s} t^{s}
\end{array}\right.
$$

avec $\forall i \in I^{\prime \prime},\left(c^{n_{q+1}^{(i)}}-\left(a_{h(S)}^{(i)}\right)^{n_{q+1}^{(i)}} \omega^{p_{q+1}^{(i)}}\right) \neq 0$.

Le $p$-uplet des valuations de $f$ le long de $\varphi$ admet alors la même valeur que dans le cas précédent et l'espace $X_{S, l}$ s'identifie alors au produit d'un espace affine 


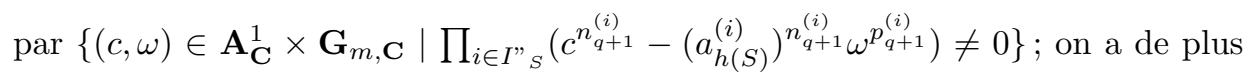
$a c(\varphi)=\overline{f "}_{S}(c, \omega)$, ce qui termine la preuve du lemme.

\subsection{Expression combinatoire de la fonction zêta d'Igusa motivique}

On a le résultat suivant :

Théorème 5.2.1. Pour tout $\alpha \in\left(\mathbf{N}^{*}\right)^{p}$ on $a$ :

$$
\lim _{T \rightarrow \infty} Z_{f}\left(T^{\alpha}\right)=-\left[\mathcal{X}_{0}\right]+\left\{\sum_{S \in I_{1}}\left[\mathcal{X}_{S}\right]+\sum_{B \in \mathcal{B}}\left[\mathcal{X}_{B}\right]\right\}-\sum_{S \in I_{2}}\left[\mathcal{X}^{\prime \prime}\right] .
$$

Preuve. Chaque espace d'arcs $X_{n}$ (pour $\left.n \in\left(\mathbf{N}^{\times}\right)^{p}\right)$ étant une réunion disjointe de constructibles du type $X_{S, l}$, la série $Z_{f}(T)$ se décompose de manière naturelle sous la forme :

$$
\begin{aligned}
& {\left[\mathcal{X}_{0}\right] Z_{0}(T)+\sum_{S \in I_{1}}\left[\mathcal{X}_{S}\right] Z_{S}(T)} \\
& \left.+\sum_{S \in I_{2}}\left[\mathcal{X}_{S}^{\prime}\right] Z_{S}^{\prime}(T)+\left[\mathcal{X}{ }^{\prime}{ }_{S}\right] Z^{\prime \prime}{ }_{S}(T)\right)+\sum_{B \in \mathcal{B}}\left[\mathcal{X}_{B}\right] Z_{B}(T)
\end{aligned}
$$

Le calcul de la limite de ces séries est analogue à celui mené dans la preuve de la proposition 3.3.1 pour le cas d'une seule fonction; il montre que $Z_{0}\left(T^{\alpha}\right)$, $Z_{S}\left(T^{\alpha}\right), Z^{\prime \prime}\left(T^{\alpha}\right), Z_{B}\left(T^{\alpha}\right)$ et $Z_{\infty}\left(T^{\alpha}\right)$ ont pour limites respectives $-1,1,-1$, et 1 quand $T \rightarrow \infty$ et que $Z_{S}^{\prime}\left(T^{\alpha}\right)$ tend vers 0 .

\subsection{Application au calcul de la fonction zêta d'Alexander}

On déduit de ce résultat une formule exprimant la fonction d'Alexander de $f$ en termes de l'arbre des contacts.

Nous allons associer à chacun de ces sommets un $A$-cycle $\zeta_{S}$ de la manière suivante :

- Si $S$ est un sommet du type 1 , alors on pose $\zeta_{S}=\left(T^{\alpha(S)}-1\right)^{(k(S)-1)}$ avec $\alpha_{i}(S)=\tilde{\beta}_{q}$ si $i \in I_{S}$ et $\alpha_{j}(S)=C_{j}(S)$ sinon.

- Si $S$ est un sommet du type 2 , on distingue deux cas :

* Soit $I_{S}^{\prime}=\emptyset$, alors on pose ${ }^{A} \zeta_{S}=\left(T^{\lambda(S)}-1\right)^{k "(S)}\left(T^{\mu(S)}-1\right)$

$$
\left\{\begin{array}{c}
\lambda_{i} "(S)=n_{q+1}^{(i ")} \bar{\beta}_{q+1}^{(i ")} \text { si } i " \in I{ }^{\prime \prime}{ }_{S} \text { et } \lambda_{j}(S)=n_{q+1}^{(i ")} C_{j}(S) \text { sinon } \\
\mu_{i} "(S)=\bar{\beta}_{q+1}^{(i ")} \text { si } i \in I{ }^{\prime \prime}{ }_{S} \text { et } \mu_{j}(S)=C_{j}(S) \text { si } j \in J_{S} .
\end{array}\right.
$$

* Soit $I_{S}^{\prime} \neq \emptyset$, alors $\zeta_{S}=\left(T^{\lambda(S)}-1\right)^{-k "(S)}$ avec les mêmes valeurs que cidessus pour $\lambda_{i^{\prime}(S)}$ si $i \notin I_{S}^{\prime}$ et la condition $\lambda_{i^{\prime}}(S)=\frac{m^{\left(i^{\prime}\right)}}{m^{\left(i^{\prime \prime}\right)}}\left(n_{q+1}^{\left(i^{\prime \prime}\right)} \bar{\beta}_{q+1}^{\left(i^{\prime \prime}\right)}\right)$ pour $i^{\prime} \in I_{S}^{\prime}$ (la valeur précédente étant indépendante du choix de $i^{\prime \prime} \in I_{S}^{\prime \prime}$ ). 
On peut alors énoncer le résultat sous la forme suivante :

Proposition 5.3.1. La fonction zêta d'Alexander de $f$ vérifie :

$$
{ }^{A} \zeta_{f_{1}, \ldots, f_{p}}=\zeta_{0} \prod_{S \in I_{1}} \zeta_{S} \prod_{S \in I_{2}} \zeta_{S}
$$

Remarque 5.3.2. Cette formule est implicite dans [16] (cf. Appendice au Chapitre 1 et Chapitre 3 section 12).

En appliquant le théorème 5.2.1 et la proposition 4.4.1, on obtient l'expression suivante de la fonction zêta d'Alexander de $f$ :

$$
{ }^{A} \zeta_{f_{1}, \ldots, f_{p}}={ }^{A} \zeta\left(\left[\mathcal{X}_{0}\right]\right)+\sum_{B \in \mathcal{B}}{ }^{A} \zeta\left(\left[\mathcal{X}_{B}\right]\right)+\sum_{S \in I_{1}}{ }^{A} \zeta\left(\left[\mathcal{X}_{S}\right]\right)+\sum_{S \in I_{2}}{ }^{A} \zeta\left(\left[\mathcal{X}{ }^{\prime}\right]\right)
$$

On en déduit alors la formule annoncée grâce au lemme suivant :

Lemme 5.3.3. i) ${ }^{A} \zeta\left(\left[\mathcal{X}_{0}\right]\right)=\left(T_{1}^{m^{(1)}} \ldots T_{p}^{m^{(p)}}-1\right)^{-1}$.

ii) Si $S$ est un sommet de type 1 , alors ${ }^{A} \zeta\left(\left[\mathcal{X}_{S}\right]\right)=\zeta_{S}$.

iii) Si $B$ est une branche ou une feuille, alors ${ }^{A} \zeta\left(\left[\mathcal{X}_{B}\right]\right)=0$.

iv) Si $S$ est un sommet de type 2 , alors ${ }^{A} \zeta\left(\left[\mathcal{X}^{\prime}{ }_{S}\right]\right)=0$ et ${ }^{A} \zeta\left(\left[\mathcal{X}{ }^{\prime}{ }_{S}\right]\right)=\zeta_{S}$.

Preuve du lemme. Le i) est un cas particulier de la situation à croisements normaux.

Considérons le cas de la variété associée à un sommet $S$ de type 1 , au point $B$ d'une branche, ou d'une feuille. Il s'agit d'évaluer le cycle d'Alexander de la $\mathbf{G}_{m, \mathbf{C}^{-}}^{p}$ variété $\mathcal{X}_{S}$; nous pouvons plonger $\mathcal{X}_{S}$ dans $\mathbf{A}_{\mathbf{C}}^{2}$ et prolonger $\bar{f}_{S}$ en un morphisme de $\mathbf{A}_{\mathbf{C}}^{2}$ dans $\mathbf{A}_{\mathbf{C}}^{p}$; nous sommes alors ramenés à la situation d'un diviseur à croisements normaux de $\mathbf{A}_{\mathbf{C}}^{2}$ de composantes $\{\omega=0\}$ et $\left\{c=a^{(i)}\right\}_{i \in I_{S}}$; la caractéristique d'Euler de la strate $\{\omega=0\} \backslash \cup_{i \in I_{S}}\left\{c=a^{(i)}\right\}$ vaut $1-k(S)$ et les autres sont nulles, ce qui démontre le ii) en appliquant la proposition 4.2.3.

Le cas d'une branche est similaire à celui d'un sommet de type 1 pour lequel $k(S)=1$, d'où ${ }^{A} \zeta\left(\left[\mathcal{X}_{B}\right]\right)=0$.

Envisageons maintenant le cas d'un sommet du type 2. Nous pouvons nous ramener à la situation d'un diviseur à croisements normaux en effectuant l'éclatement torique associé à un raffinement régulier de l'éventail du premier cadrant d'arêtes $\left\{(1,0),\left(p_{q+1}, n_{q+1}\right),(0,1)\right\}$. Nous devons distinguer deux cas :

- Soit $I_{S}^{\prime}=\emptyset$, la transformée totale de $\bar{f}^{\prime \prime}{ }_{S}=0$ est la réunion des composantes du diviseur exceptionnel associées aux arêtes de coordonnées strictement positives et de la transformée stricte de $\omega=0$ (qui correspond à l'arête $(1,0)$ ). Chaque composante en intersecte deux autres excepté le premier "bout", qui en intersecte un seul, le diviseur de rupture qui en intersecte deux outre les transformées strictes des fonctions $\left(c^{n_{q+1}}-\left(a_{r_{q+1}}^{(i)}\right)^{n_{q+1}} \omega_{l} p_{q+1}\right)_{i \in I{ }^{\prime}}$ et la transformée stricte de $\omega=0$ qui en intersecte un seul (le deuxième "bout"). Le nombre de transformées strictes 
ci-dessus est aussi égal au nombre de branches du diagramme des contacts qui se séparent au niveau du sommet $S$, c'est-à -dire à $k "(S)$.

Les multiplicités de $c^{n_{q+1}}-\left(a_{r_{q+1}}^{(i)}\right)^{n_{q+1}} \omega_{l} p_{q+1}$ pour $i \in I{ }_{S}$, (resp. $\omega$ ) le long du diviseur de rupture valent $n_{q+1} p_{q+1}$ (resp. $n_{q+1}$ ) et les multiplicités le long du bout valent $n_{q+1}($ resp. 1$)$; le premier cas du $\left.i v\right)$ s'en déduit puisque

$$
n_{q+1} p_{q+1} e_{q+1}+n_{q+1} \tilde{\beta}_{q}=n_{q+1} \bar{\beta}_{q+1} .
$$

- Dans le cas où $I_{S}^{\prime} \neq \emptyset$, la transformée totale de $\bar{f}_{S}=0$ contient une composante supplémentaire (la transformée totale de $c=0$ ) qui intersecte le premier "bout". Le seul diviseur dont la contribution à la fonction zêta d'Alexander est éventuellement non nulle est donc le diviseur de rupture; la multiplicité de $c$ le long de ce diviseur vaut $p_{q+1}$; pour tout $i^{\prime} \in I_{S}^{\prime}$, l'ordre de la fonction $c^{e_{q}^{\left(i^{\prime}\right)}} \omega^{\tilde{\beta}_{q}^{\left(i^{\prime}\right)}}$ vaut $p_{q+1}^{\left(i^{\prime \prime}\right)} e_{q}^{\left(i^{\prime}\right)}+n_{q+1}^{(i ")}\left(\bar{\beta}_{q+1}^{\left(i^{\prime}\right)}-\beta_{q+1}^{\left(i^{\prime}\right)}\right)\left(\right.$ pour $i "$ quelconque dans $I^{\prime \prime}{ }_{S}$ et $\left.i^{\prime} \in I_{S}^{\prime}\right)$ c'est-à-dire

$$
\frac{m^{\left(i^{\prime}\right)}}{m^{\left(i^{\prime \prime}\right)}}\left(n_{q+1}^{(i ")} \bar{\beta}_{q+1}^{(i ")}\right)=: \lambda_{i^{\prime}}(S)
$$

comme annoncé. Ceci achève le calcul et la démonstration de la proposition 5.3.1.

$\diamond$ Exemple 1 : Dans le cas d'une seule fonction, chaque paire de Puiseux donne lieu à l'apparition d'un sommet $S$ qui est de type 2 et pour lequel $I_{S}^{\prime}=\emptyset$. La formule 5.3.1 s'écrit donc

$$
{ }^{A} \zeta_{f}=\left(T^{m}-1\right)^{-1} \prod_{q=1}^{g} \frac{\left(T^{n_{q} \bar{\beta}_{q}}-1\right)}{\left(T^{\bar{\beta}_{q}}-1\right)}
$$

qui est bien l'inverse de la fonction zêta de la monodromie de $f$ (voir corollaire 3.5.1).

$\diamond$ Exemple 2 : Considérons le cas où

$$
f_{1}(x, y)=y^{2}-x^{3} \quad \text { et } \quad f_{2}(x, y)=\left(y^{2}-x^{3}\right)^{2}-4 y x^{5}-x^{7}
$$

Nous avons pour la première fonction : $m=2, \beta_{1}=3, e_{1}=1, p_{1}=3, n_{1}=2$.

Pour la deuxième, on a : $m=4, \beta_{1}=6, e_{1}=2, p_{1}=3, n_{1}=2 ; \beta_{1}=7$, $e_{2}=1 ; \bar{\beta}_{2}=13$.

Les deux développements fractionnaires associés à $f_{1}$ sont $x^{\frac{3}{2}}$ et $-x^{\frac{3}{2}}$; ceux associés à $f_{2}$ sont $x^{\frac{3}{2}}+x^{\frac{7}{4}}, x^{\frac{3}{2}}-x^{\frac{7}{4}},-x^{\frac{3}{2}}+i x^{\frac{7}{4}}$ et $-x^{\frac{3}{2}}-i x^{\frac{7}{4}}$.

Le diagramme des contacts de $f_{1}$ et $f_{2}$ admet deux sommets $S_{0}$ et $S_{1}$, le premier est d'altitude $\frac{3}{2}$ et le second d'altitude $\frac{7}{4}$.

Le sommet $S_{0}$ est de type 2 et on a $I_{S_{0}}^{\prime}=\emptyset$ et $k^{\prime \prime}\left(S_{0}\right)=2$, d'où $\zeta_{S_{0}}\left(T_{1}, T_{2}\right)=$ $\left(T_{1}^{12} T_{2}^{12}-1\right)^{2}\left(T_{1}^{6} T_{2}^{6}-1\right)^{-1}$.

Le sommet $S_{1}$ est de type 2 ; on a $I_{S_{1}}^{\prime}=\{1\}, k^{\prime}\left(S_{1}\right)=1$ et $k^{\prime \prime}\left(S_{0}\right)=1$, d'où $\zeta_{S_{1}}\left(T_{1}, T_{2}\right)=\left(T_{1}^{26} T_{2}^{26}-1\right)$.

Il vient finalement

$$
{ }^{A} \zeta_{f_{1}, f_{2}}\left(T_{1}, T_{2}\right)=\left(T_{1}^{2} T_{2}^{4}-1\right)\left(T_{1}^{12} T_{2}^{12}-1\right)^{2}\left(T_{1}^{6} T_{2}^{6}-1\right)^{-1}\left(T_{1}^{26} T_{2}^{26}-1\right) .
$$




\subsection{Application au calcul de la fibre de Milnor motivique d'une série de deux variables éventuellement réductible}

On s'intéresse au calcul de la fibre de Milnor motivique du produit $F:=f_{1} \times$ $\ldots \times f_{p}$. On dispose d'une application naturelle $m: \mathbf{G}_{m, \mathbf{C}}^{p} \rightarrow \mathbf{G}_{m, \mathbf{C}}$ définie par $\left(t_{1}, \ldots, t_{p}\right) \mapsto t_{1} \ldots t_{p}$.

Cette application induit de manière naturelle une application $m_{*}$ entre $K_{0}\left(\mathbf{G}_{m, \mathbf{C}}^{p}\right)$ et $K_{0}\left(\mathbf{G}_{m, \mathbf{C}}\right)$ qui s'étend de manière naturelle en une application qu'on note encore $m_{*}$ entre

$$
\mathcal{M}_{\mathbf{G}_{m, \mathbf{C}}^{p}}\left[\left[T_{1}, \ldots, T_{p}\right]\right] \text { et } \mathcal{M}_{\mathbf{G}_{m, \mathbf{C}}}[[T]]
$$

définie par

$$
m_{*}\left([\mathcal{X}] T_{1}^{\nu_{1}} \ldots T_{p}^{\nu_{p}}\right):=m_{*}([\mathcal{X}]) T^{\nu_{1}} \ldots T^{\nu_{p}}
$$

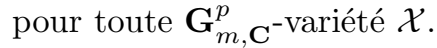

Il résulte directement des définitions qu'on a la relation $Z_{F}=m_{*}\left(Z_{f}\right)$ et on déduit alors du théorème 5.2.1 la proposition suivante :

Proposition 5.4.1. La fibre de Milnor motivique de la série $F$ s'exprime à l'aide de l'arbre des contacts par la relation suivante:

$$
\mathcal{S}_{F, 0}=\left[\mathcal{Y}_{0}\right]+\left\{\sum_{S \in I_{1}}\left[\mathcal{Y}_{S}\right]+\sum_{B \in \mathcal{B}}\left[\mathcal{Y}_{B}\right]\right\}+\sum_{S \in I_{2}}\left[\mathcal{Y}^{\prime \prime}\right]
$$

où les notations sont les suivantes :

- $\mathcal{Y}_{0}:=\left\{x \in \mathbf{G}_{m, \mathbf{C}} \mid x^{m}=1\right\}$ (avec $m:=\sum_{i} m^{(i)}$ ) munie de l'action de $\mu_{m}$ définie par $\zeta . x:=\zeta x$.

- Pour tout $S$ sommet du type 1, branche de l'arbre des contacts, ou feuille

$$
\mathcal{Y}_{S}:=\left\{(x, y) \in \mathbf{A}_{\mathbf{C}}^{1} \times \mathbf{G}_{m, \mathbf{C}} \mid \prod_{i \in I_{S}}\left(x-a_{h(S)}^{(i)}\right)_{q}^{e_{q}^{(i)}} y^{\sum_{i \in I_{S}} \tilde{\beta}^{(i)}(S)}=1\right\}
$$

où l'on a noté $\tilde{\beta}^{(i)}(S)=\tilde{\beta}_{q(S)}^{(i)}$ si $i \in I_{S}$ et $C_{S}(i)$ sinon. Si $N_{q(S)} h(S)=p / n$ avec $p$ et $n$ premiers entre eux, l'action de $\hat{\mu}$ sur $\mathcal{Y}_{S}$ se factorise par l'action de $\mu_{n n(h(S)}$ définie par $\zeta .(x, y):=\left(\zeta^{p} c, \zeta^{n} y\right)$.

- Pour tout $S$ sommet du type 2 ,

$$
\begin{aligned}
\mathcal{Y}_{S}:= & \left\{(x, y) \in \mathbf{A}_{\mathbf{C}}^{1} \times \mathbf{G}_{m, \mathbf{C}} \mid\right. \\
& \left.\prod_{i \in I_{S}^{\prime \prime}}\left(x^{n_{q+1}}-a_{r_{q+1}}^{(i)} y^{p_{q+1}}\right)^{e_{q+1}} x^{\sum_{i \in I_{S}^{\prime}} e_{q}^{(i)}} y^{\sum_{i} \tilde{\beta}_{q}^{(i)}}=1\right\}
\end{aligned}
$$

où $\tilde{\beta}_{q}^{(i)}$ a la même signification que ci-dessus. L'action de $\hat{\mu}$ sur $\mathcal{Y}{ }_{S}$ se factorise par l'action de $\mu_{n_{q+1} \bar{\beta}_{q+1}}$ définie par $\zeta .\left(c, \omega_{l}\right):=\left(\zeta^{p_{q+1}} c, \zeta^{n_{q+1}} \omega_{l}\right)$. 


\section{Références}

[1] N. A'Campo, La fonction zêta d'une monodromie, Comment. Math. Helv. 50 (1975), 233248.

[2] V. Batyrev, Birational Calabi-Yau $n$-folds have equal Betti numbers, in : Klaus Hulek et al. (eds.), New trends in algebraic geometry, 1-11, CUP, 1999.

[3] J. L. Brylinski, Transformations canoniques, dualité projective, geometrie et analyse microlocale, Astérisque 140-141 (1986), 3-134

[4] A. Campillo, F. Delgado et S. M. Gusein-Zade, On the monodromy of a plane curve singularity and the Poincaré series of its ring of functions, Funct. Analysis and its Appl. $\mathbf{3 3}$ $66-68$.

[5] A. Campillo, F. Delgado et S. M. Gusein-Zade, Integration with respect to the Euler characteristic over a function space and the Alexander polynomial of a plane curve singularity, Russian Mathematical Surveys 55 (2000), 6.

[6] P. Deligne et N. Katz, SGA7. Groupes de monodromie en géométrie algébrique, Lect. Notes in Math. 340 (1972-1973).

[7] J. Denef, Report on Igusa's local zeta function, Séminaire Bourbaki 1990/1991, exposé 741. Astérisque 201-202-203 (1991), 359-386.

[8] J. Denef, Degree of local Igusa function and monodromy, Compositio Mathematica 89 (1993), 207-216.

[9] J. Denef et K. Hoornaert, Newton polyhedra and Igusa' s local zeta function, Journal of number Theory (à paraitre).

[10] J. Denef et F. Loeser, Caractéristique d'Euler-Poincaré, fonctions zêta locales et modifications analytiques, J. Amer. Math. Soc. 5 (1995), 705-720.

[11] J. Denef et F. Loeser, Motivic Igusa zeta functions, Journal of Algebraic Geometry 7 (1998), 505-537.

[12] J. Denef et F. Loeser, Germs of arcs on singular algebraic varieties and motivic integration, Inventiones Math. 135 (1999), 201-232.

[13] J. Denef et F. Loeser, Motivic exponential integrals and a motivic Thom-Sebastiani theorem, Duke Math. J. 99 (1999), 285-309.

[14] J. Denef et F. Loeser, Lefschetz numbers of the monodromy and truncated arcs, arXiv.org/abs/math/0001105.

[15] J. Denef et F. Loeser, Geometry on arc spaces of algebraic varieties, arXiv.org/abs/ math/0006050.

[16] D. Eisenbud et W. Neumann, Three-dimensionnal link theory and invariants of plane curve singularities, Ann. of Math. Studies 110, Princeton Univ. Press, Princeton, NJ, 1985.

[17] M. Kontsevitch, Lecture at Orsay, 1995.

[18] A. G. Kouchnirenko, Polyèdres de Newton et nombres de Milnor, Invent. Math. 32 (1976), $1-32$.

[19] D. T. Lê, Sur les noeuds algébriques, Compositio Math. 25 (1972), 281-321.

[20] E. J. N. Looijenga, Motivic measures, Séminaire Bourbaki, Exposé 874, Mars 2000.

[21] C. Sabbah, Modules d' Alexander et D-modules, Duke Math. J. 60 No. 3 (1990).

[22] M. Saito, Exponents of an irreducible plane curve singularity, arXiv.org/abs/math/0009133.

[23] J. Steenbrink, Mixed Hodge structure in the vanishing cohomology, in : P. Holm, ed., Real and Complex singularities, 525-563, Sijthoff and Noordhoff, Oslo, 1977. 
[24] J. Steenbrink, The spectrum of hypersurface singularities, Theorie de Hodge, Luminy 1987, Asterisque 179-180 (1989), 163-184.

[25] O. Zariski, Le problème des modules pour les branches planes, Centre de Mathématiques de l'École polytechnique, 1973, réédition Hermann, 1986.

Gil Guibert

Laboratoire J. A. Dieudonné

UMR CNRS 6621

Université de Nice-Sophia Antipolis

Parc Valrose

06108 Nice Cedex 2

France

(Received: February 15, 2002)

(1) To access this journal online:

(4) http://www.birkhauser.ch 